\title{
ELECTRON MICROSCOPY STUDY OF MICROSEGREGATION AND DEFECTS IN CZOCHRALSKI GROWN CALCIUM GALLIUM GERMANIUM GARNET SINGLE CRYSTALS
}

Thomas Franklin Roth

(M. S. thes is)

September 1978

$$
\begin{aligned}
& \text { 2.5 C E IV }
\end{aligned}
$$

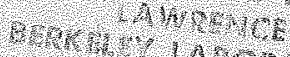

$$
\begin{aligned}
& \text { HURMGY AND } \\
& \text { notiments Srot }
\end{aligned}
$$

Prepared for the U. S. Department of Energy under Contract W-7405-ENG-48

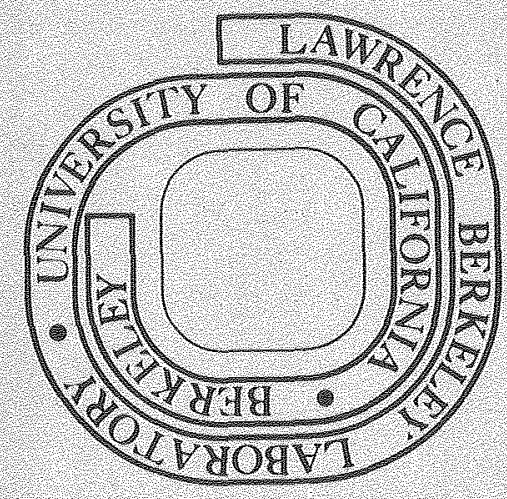

TWO-WEEK LOAN COPY

This is a Library Circulating Copy which may be borrowed for two weeks. For a personal retention copy, call Tech. Info. Dívision, Ext. 6782 


\section{LEGAL NOTICE}

This report was prepared as an account of work sponsored by the United States Government. Neither the United States nor the Department of Energy, nor any of their employee or any of their contractors, nimantractors, or their employ' $\alpha_{e}^{\prime} \quad$ kes any warranty, express o $\quad 1$, or as ines any legal lis, the accuri ipletent fuiness of ratus, pre not infri rocess 1, or represe isponsibility for rmation, appa$\checkmark \mathrm{C}$ in its use would 
ELECTRON MTCROSCOPY STUDY OF MICROSEGREGATION AND DEFECTS IN CZOCHRALSRI GROWN CALCIUM GALLIUM GERMANIUM GARNET SINGLE CRYSTALS

Thomas Frank1 in Roth

Lawrence Berkeley Laboratory

Bexkeley, California 94720 

ELECTRON MTCROSCOPY STUDY OF MTCROSEGREGATION AND DEFECTS IN CZOCHRALSRI GROWN CALCIUM GALLIUM GERMANIUM GARNET SINGLE CRYSTALS

\section{Contents}

Abstract . . . . . . . . . . . . . . . . . . iv

I. Introduction ................. 1

II. The Garnet Structure . ............ 5

III. Specimen Preparation .............. 11

A. Czochralski Grown CGGG Single Crystals....... 11

B. Transmission Electron Microscopy Specimen

Preparation.................. 13

IV. Electron Diffraction and Imaging......... 15

V-A. Microsegregate Defects/Results......... 19

V-B. Microsegregate Defects/Discussion ........ 25

VI-A. Chemical Analysis/Results ............ 32

VI-B. Chemical Analysis/Discussion........... 34

VII-A. Dislocations/Results ............40 40

VII-B. Dislocations/Discussion.............44 43

VIII-A. Coherenc Precipikates/Results . . . . . . . . 49

VII-B. Coherent Precipitates/Discussion ......... 51

IX. Model For Microsegregate Formation....... 57

Conclusions . . . . . . . . . . . . . . . 62

Suggestions for Further Study .................63 63

Acknowledgements .................... 64

Appendis/Chemical Analysis ............... 66

References....................... 71

Figure Captions . . . . . . . . . . . . . . . 74

Figures .......................... 82 

crystallographic directions although arrays along $<110>$ have also been observed.

Several configurations of dislocations in close proximity to the segregate inhomogeneities were common. Despite their physical association with the microsegregates the dislocations are always found to lie in the matrix material and never internal to the microsegregated regions. Some dislocation lines lying strictly in the matrix were found to have Burgers vectors parallel to $<113>$ directions. Dislocation lines lying at the matrix/microsegregate interface or lying in the matrix but in contact with this interface are also common and are confirmed to have Burgers vectors parallel to $<112>$ crystallographic directions. None of these dislocations showed evidence of dissociation into partial dislocations and are consequently assumed to be perfect.

Dislocation loops lying in the matrix and encircling the microsegregate defects are also observed. These loops are typically about $0.6 \mathrm{H}$ in diameter. The loops are found to be of edge type having Burgers vectors parallel to $<100>$ directions and perpendicular to the $\{100\}$ planes on which the loops lie. Evidence of dissociation of these loops into partial dislocations whose Burgers vectors are not parallel to $<100>$ directions has been found.

In order to detemine the origin of the microsegregate defects and the various dislocation configurations requires understanding the processing history of the Czochralski grown CGGG single crystal boule in conjunction with these microscopy results. The chemical inhomogeneities result from evaporation of germanium from the crystal growth melt and segregation of gallium, which accumulates locally, 
in the growing crystal. The evaporation and segregation processes are suggested to be sensitive to thermal fluctuations and gradients in the growth melt as well as processing parameters such as melt composition, growth atmosphere, crystal rotation rate, and crystal pulling rate. 


\section{Introduction}

Much work is currently being done on solid state computer memory elements in which binary information is stored in cylindrical magnetic domains, ofren called magnetic bubbles. 1,2 Magnetic bubble domains can be supported in thin erystals displaying uniaxial magnetic anisotropy perpendicular to the plane of the crystal. These bubbles can be manipulated via applied magnetic fields and made to translate predictably and reliably within the crystal. This domain mobility, in conjunction with the ability to generate, detect, and annihilate bubbles at specified locations on a Eabricated bubble memory chip, provides the computer functions of writing, retrieving, and reading information. Since the magnetic domain is the only moving enticy in such an information storage scheme, bubble devices do not suffer mechanical wear problems as do conventional memory systems such as tapes or disks. To obtain economically competitive information storage densities $\left(-10^{6}\right.$ bits $\left./ \mathrm{cm}^{2}\right)$ very thin $(-3 \mu)$ magnetic crystals are required so that small diameter $(\sim 2, \mu)$ bubbles are statically and dynamically stable. ${ }^{3}$

Thin films of ferrimagnetic garnets are prime candidates for these bubble merory devices because garnets are found to support bubbles of small enough size and sufficient mobility to provide attractive data storage densities and information retrieval rates. ${ }^{4}$ For such applications, single crystal magnetic garnet films are grom on nonmagnetic garnet substrates through a liquid-phase epitamial growth process. The single-crystal substrate not only provides nucleation sites for the growth of the epitaxial film but gives mechanical 
strength to the thin and Fragile epitaxial layer in a device chip. In addition, the lactice constants of the substrate and epitaxial garnets are controlled such that there is a slight lattice parameter mismatch which results in a stressed induced component to the uniaxial. magnetic anisotropy of the epitaxial layer. 5

Bubble device performance is dixectly related to the uniformity and perfection of the magnetic film. Static and dynamic properties of bubble domains are altered by magnetic defects in the film. A magnetic defect is a perturbation in the periodic magnetic spin arrangement in the matexial. The presence of crystal defects implies a discontinuity in the magnetic moment configuration since the forces between atoms in crystals are significantly greater than the forces among spins which maintain their alignment. ${ }^{6}$ Thus crystal defects can act as sites for undesirable domain pinning or erratic dynamic behavior. Consequently a defect-free magnetic film is desired.

A prerequisite for the growth of a defect-free film is a defectEree substrate. ${ }^{3}$ Duxing the liquid-phase epitaxial growth of the magneric film on the substrate, any substrate defect which intersects the substrate surface can act as a preferential nucleation site for the magnetic phase. In the case of a substrate dislocation, the dism ordered core region of the dislocation can permit accomodation of growth units of the magnetic phase in configurations not normally found at undefective (homogeneous) nucleation sites. In this way substrate dislocations can be extended into the epicaxial layer ${ }^{7,8}$ with resulting degradation of crystal perfection and device performance. Thus, to insure reliable operation of bubble device chips they rust 
be fabricated so as to produce relatively defect-free magnetic thin films and this in curn requires a virtually defect-free substrate.

Such garnet substrates have usually been made from gadolinium gallium garnet ( $G G G$ or $\mathrm{Gd}_{3} \mathrm{Ga}_{5} \mathrm{O}_{12}$ ). However, recently attencion has been dram to calcium gallium gexmanium garnet ( $C G G G$ or $\mathrm{Ca}_{3} \mathrm{Ga}_{2} \mathrm{Ge}_{3} \mathrm{O}_{12}$ ) because of its superiority over GGG with respect to cost and ease of processing. 9 The relarively low melting temperature of $1380^{\circ} \mathrm{C}$ for CGGG allows Czochralski boules to be grown from flux melts at a growth temperature about $350^{\circ} \mathrm{C}$ lower than that for GGG. This permits the use of less expensive platinum (instead of ixidium) crucibles. Also, in the CGGG growth process the lower heat input for melting requires one third of the electric power and correspondingly less cooling water than GGG crystal growth. Other comercial advantages arise since GGG is harder than CGGG, and thus the cutting, grinding, and polishing of CGGG substrate wafers from the boules is more easily accomplished. Calcium is also more abudant and cheaper than gadolinium (as is germanium relative to gallium), making CGGG less expensive than GGG. However the smallex lattice parameter of CGGG relative to that for GGG would necessitate development of new ferrimagnetic epi-layer garnet compositions to give the desixed lattice parameter mismatch and consequent stress induced magnetic anisotropy needed for device applications.

The CGGG single crystal boules have been grown with relative success using knowledge obtained from the extensive research on GGG crystal growth. At this time, however, defect densities in CGGG are higher than those obtained in GGG crystals. It is hoped that characterization 
of tnicrostructural defects in CGG will suggest ways to optimize processing parameters in order to eliminate the defects. For this reason the objective of this research is to systematically study and characterize microstructural defects in CGGG using electron microscopy cechniques.

In the present research two major classes of defects were observed which were distinctly different yet still related. Chemical inhomogeneities in the garnet matrix material were identified and characterized with respect to their distribution, structure, morphology, crystallography, and composition. Associated with these chemical defects were various configurations of dislocations which were characterized with respect to their type, distribution, Burgers vectors, and habit plane (when this was possible).

This work presents the experimental methods and results of these defect characterizations. The results of this study are significant for two reasons. First, microstructure/processing correlations will have significant impact on the applicability of CGGG in bubble devices. Second, a survey of the literature reveals little published work on systematic defect characterigations in garnet systems at electron microscope resolutions. Consequently the microscopic nature of garnet defects remains virtually unknown. In short, this work is not only of contemporary comercial interest but is also of fundanental academic concern. 


\section{The Garnet Structure}

Investigating defects in garnet materials requires familiarity with the garnet structure. The general chemical formula of garnets is $\mathrm{M}_{3} \mathrm{M}_{2} \mathrm{M}_{3}^{\prime \prime} \mathrm{O}_{12}$ where $\mathrm{M}, \mathrm{M}^{\prime}$, and $\mathrm{M}^{\prime \prime}$ are metallic cations. In many of the synthetic garnets the metal ions are all trivalent (i.e.+3 charge in $\left.\mathrm{Gd}_{3} \mathrm{Ga}_{5} \mathrm{O}_{12}\right)$. CGG is like the natural garnets in which the cations are of varying size with ionic radi $r$ and occur in different valence states as follows:

$\begin{array}{lll}\mathrm{Cg} & +2 & \mathrm{r}=0.99 \mathrm{~A} \\ \mathrm{Ga} & +3 & \mathrm{r}=0.62 \mathrm{~A} \\ \mathrm{Ge} & +4 & \mathrm{r}=0.53 \mathrm{~A} \\ \mathrm{co} & -2 & \mathrm{r}=1.40 \mathrm{~A}\end{array}$

Scoichiometry and electroneutrality are maintained by substicucion of a +2 and $a$ th ion paix for each pair of +3 ions replaced.

The garnet structure belongs to space group $0_{h}^{10}$-Ia3d having a unit cell showing body centered cubic symetry with a typically large latrice parametex $\left(a_{0}=12.252\right.$ in $\left.C G G G\right) .10$ The unit cell contains eight $\mathrm{Ca}_{3} \mathrm{Ga}_{2} \mathrm{Ge}_{3} \mathrm{O}_{12}$ formula units, giving a cotal of $8 \times 20=160$ ions per unit cell. Of these 160 ions, 96 are oxygen and it is tempting to describe the structure in terms of the oxygen sublattice. However the oxygens are arranged in neither a simple nor close-packed manner and it is more convenient to vigualige their distribution in terms of cation polyhedra. ${ }^{11}$ The M cations axe surrounded by eight oxygen anions in dodecahedral coordination. The M cation lattice positions are referred to as $c$ sites. A dodecahedron is a distorted cube (Fig. $\mathrm{A}$ ) and c sices prowide the least dense packing of omygen. 
Consequently the largest cations usually occupy c sites (calcium in CGGG). The $M^{8}$ sites are the most densely packed with six-fold coordination of oxygen in octahedral symmetry (Fig.1B). These are called a sites and they accomodate small metal carions ( $\mathrm{Ga}$ ) because of the dense oxygen packing. The $\mathrm{M}^{\mathrm{B}}$ sites are surrounded by four oxgen atoms in retrahedral coordination (Fig. 1C) and are called d sites. The d sites have an oxygen packing density intermediate to the a and $c$ sites and are occupied by Ge ions in CGGG.

Each oxygen is shared by four polyhedra (one tetra-, one octa-, and two dodecahedra) as is seen in Fig. 2. The edge lengths of any of the polyhedxa are not all equal but the axygen-cation separation is about equal in each case. 11 The positions of the ions in the unit cell as given by Wykoff ${ }^{12}$ are as follows:

$$
\begin{aligned}
& \mathrm{Ca}: \quad(24 \mathrm{c}) \pm(1 / 801 / 4 ; 1 / 41 / 80 ; 01 / 41 / 8 ; 5 / 801 / 4 ; \\
& 1 / 45 / 80 ; 01 / 45 / 8) ; B . C \text {. } \\
& \text { Ga: (16a) } \quad(000 ; 1 / 21 / 20 ; 01 / 21 / 2 ; 1 / 201 / 2 ; \\
& 1 / 41 / 41 / 4 ; 3 / 43 / 41 / 4 ; 1 / 43 / 43 / 4 ; \\
& 3 / 41 / 43 / 4) ; B . C \text {. } \\
& \text { Ge: } \quad(24 \mathrm{~d}) \div(3 / 801 / 4 ; 1 / 43 / 80 ; 01 / 43 / 8 ; 7 / 8 \quad 0 \quad 1 / 4 ; \\
& 1 / 47 / 80 ; 01 / 47 / 8) ; B . C \text {. } \\
& 0: \quad(96 \mathrm{H}) \pm\left(\mathrm{xyz} z \mathrm{z}+1 / 2,1 / 2-\mathrm{y}_{\mathrm{z}}, \overline{\mathrm{z}} ; \overline{\mathrm{x}}, \mathrm{y}+1 / 2,\right. \\
& 1 / 2-z ; 1 / 2-x_{1}, \bar{y}, z+1 / 2 ; y+1 / 4, \\
& x+1 / 4, z+1 / 4 ; y+3 / 4,1 / 4-x, \\
& 3 / 4-z ; 3 / 4-y, x+3 / 4,1 / 4-z ; \\
& 1 / 4-y, 3 / 4-x, z+3 / 4) ; E x ; B . C .
\end{aligned}
$$


The designation $B$. C. indicates that a body center cranslation of $1 / 2$, $1 / 2,1 / 2$ will generate aditional lattice points. The oxygen parameters are not rigorously established for CGGG but in other garnets they tend to have values of $x=0.04, y=0.05$, and $z=0.65$.

The structure is more easily visualized by considering the unit cell to be made up of eight octants, each octant containing one $\mathrm{M}_{3} \mathrm{M}_{2} \mathrm{M}_{3} \mathrm{O}_{12}$ formula unit. Considering only one cubic octant, the structure of each octant can be generated as follows: 1) put octahedral (a) sites in a body centered cubic configuration as shown in Fig. 3A; 2) draw non-diagonal bisectors on each of the three mutually perpendicular $\{100\}$ faces of the octant such that the three bisectors are also mutually. perpendicular, i.e. parallel to $<100>($ ig. 3B); 3) alternately distribute cerrahedral (d) and dodecahedral (c) sires along these bisectors so as to define a three-fold axis in the octant as shown in Fig. 3C.

This gives the cation configuration of each octant. The eight octants are then distributed in the unit cell so that the arrangement of three-fold axes is as shom in Fig. 4A. This distribution of threefold axes is obtained by treating each of the face bisectors of the octant (Fig. 4b) as a two-fold axis of rotation. By choosing an arbitrary octant as reference and rotating it by $180^{\circ}$ about the two-fold face bisectors, the unit cell can be completely generated as in Fig. $4 \mathrm{C}$. The bonding in garnets is mostly ionic with some covalent character as is partially reflected in the fact that the carion polyhedra share edges and vertices but not races. Geller and Gilleo ${ }^{13}$ refer to the garnet structure as racher loose since the forces mantaining it are relatively weak and not colerant of large distortions. 
A treatment of crystal defects in the garnet structure must account for the energies associated with the defects. The orderly arrangement of cations in the garnet structure leads to the possibility of many permutations in this order. Variations in the orderly distribution of cations could result from the dissociation of dislocations with faults created. This could lead to a situation in garnets similar to that found in some less complex b.c.c. alloys were dislocation interactions are not solely governed by minimizing the Burgers vector. Instead dissociated dislocations with larger Burgers vectors are observed due to the consequent decrease in fault energies. 14

Atomic level considerations of fault energies must include not only the electostatic energy resulting fxom oxygen displacements but also the changes in anion-cation bond energy associated with the altered cation and anion sublattices. It is reasonable to suggest that the lowest energy defects will be those which leave the least number of ions displaced from their lattice sites. Since the orygen packing is always disturbed by defects such as dislocations, to a first approximation in garnets the least energy will be associated with defects which leave the most densely packed (octahedral) sublattice unaltered. Defects which leave the b.c.c. sublattice unfaulted result in perturbacions of the oxygen packing which can be accomodated by small rotations of oxygen octahedra near the fault plane. For these reasons this discussion of garnet defects is limited to those leaving octahedral sites conserved.

The shortest translation vector of the Bravais lattice is $\left(a_{0} / 2\right) K 111>$ and is thus suspect as a Burgers vector of dislocations 
in garnets. Since the latrice parameter of CGGG is $12.252 \AA$ a Burgers vector of $\left(a_{0} / 2\right)\langle 11>$ is relatively large with length of $|\vec{b}|=(\sqrt{3} / 2) a_{0} \approx 10.61 \AA$. Consequently a lowering of energy might be expected from the dissociation of a $\left(a_{d} / 2\right)<11>$ perfect dislocation. If the resulting partial dislocations were split with a stacking fault in between, then depending on the energy of the fault, other dislocation dissociations could be energetically favorable also. For example, the elastic energies associated with $\left(a_{0} / 2\right)<111>$ and $a_{0}<001>$ Burgers vectors are not very different $\left(0.75 \mathrm{a}_{0}^{2}\right.$ and $a_{0}^{2}$ respectively). Depending on the energy of a stacking fault bounded by partials from a dissocated a $<001>$ dislocation, the splitting of these dislocations may be energetically favored over dissociation of perfect dislocations with Burgers vectors of $\left(a_{0} / 2\right)<111>_{0} 15$

This discussion assumed that the fault energies associated with such dissociations axe not equivalent and it is likely that they are appreciably different. Consider Fig. 5A-C which schematically represent portions of a faulted garnet unit cell with displacenent vectors $R_{a}=\left(a_{0} / 2\right)<100>, R_{b}=\left(a_{0} / 2\right)<110>$, and $R_{c}=\left(a_{0} / 4 k 111>\right.$ respectively. Inspection of these figures shows that the orderly distribution of the tetrahedral and dodecahedral sites is disrupted by each of these displacements, which could result from the dissociation of perfect dislocations. It can also be seen that each kind of defect leaves the octahedral sublatcice unaltered in accordance with the basic assumption. However, an important difference can also be seen.

The displacements $\left(a_{0} / 2\right)<100>$ and $\left(a_{0} / 2\right)<110>$ of $F i g s .5 A$ and $5 B$ are equivalent to exchanging positions of tetrahedral and 
dodecahedral cations. The octahedral ( $\left.\mathrm{Ga}^{3+}\right)$ and tetrahedral $\left(\mathrm{Ge}^{4+}\right)$ ions in CGGG are of similar size but vary in valence. Dislocations having Burgers vectors of $\left(a_{0} / 2\right)<100>$ and $\left(a_{0} / 2\right)<110$ - would therefore be expected to have electrostaric as well as elastic contributions to their rotal energy.

On the other hand, Fig. $5 \mathrm{C}$ illustrates that a displacement of $\left(a_{0} / 4\right)<111>$ does not mexely result in an exchange of lattice sices. Such a displacement will cause initially empty sites in the regular garnet lactice to be filled and cations bounding the fault plane will be brought into much closer proximity. Such displacements can be visualized as a non-stoichiometric addition or removal of a crystal slice, and the energy associated with such a defect will have an appreciably different electrostatic contribution than that of a defect causing only disordering of the cation sites.

The differences between electrostatic and strain energies associated with the various defect displacements promote a variety of possible perfect and dissociated garnet dislocations. Experimental observations of splitting widths of garnet stacking faults with definitive Burgers vector determinations could give considerable insight into the rypes of atomic level interactions which influence the existence of particular defect configurations. 


\section{Specimen Preparation}

A. Czochralski Grown CGGG Single Crystals

The CGGG single crystals used in this study were provided by Philips Laboratories of Eindhoven, Netherlands. Crystal growth procedures were as follows. 16 Powders of $99.99 \%$ puriky $\mathrm{CaCO}_{3}$, $\mathrm{Ga}_{2} \mathrm{O}_{3}$, and $\mathrm{GeO}_{2}$ were obtained From Merck (Damstadt, Germany), Alusuisse (Neuhausen, Switgerland), and Hoboken (Hoboken, Belgium) respectively. The starting powder mixture had composition $\mathrm{Ca}: \mathrm{Ga}$ : Ge=73.2:24.8:38 atomic percent. Three hundred (300) grams of the nixture was homogenized with $120 \mathrm{ml}$ of ethanol in an agate ball mill for 30 minutes. Cylindrical tablets $50 \mathrm{~mm}$ in dianeter were pressed from the dried mixture and fired 16 hours at $900^{\circ} \mathrm{C}$ to expel carbon dioxide and volarile ingurities.

Fired tablets for the Crochralski growth melt were placed in a platinum crucible 50 high, 50 mo internal diameter, and with 1 mm thick wals. The r.k. heated crucible was insultated with 3 mo zirconia felt and 5 molumina. Covering the crucible was a lid with a 25 mon diameter hole at its center. The powder mixture was heated and equilibrated near $1380^{\circ} \mathrm{C}$ (the melting point of CGGG).

Isothermal growth of Czochralski single erystals was accomplished using a rotation rate of 55 RPM and a pulling rate of $5 \mathrm{~mm}$ per hour. Cooling to room temperature arter completion of crystal growth was done in two hours.

Two complications were incurred during the growth of the CGGG single crystals. ${ }^{9}$ An appreciable amount of evaporation occurred, forming white deposit on the pulling shaft. The deposit was determined 
to be pure $\mathrm{GeO}_{2}$ by $x$-ray diffraction. As a result of the evaporative loss of Ge the composition of the growth melt changes from its original ratio via $\mathrm{Ca}: \mathrm{Ga}: \mathrm{Ge}=37.5: 25: 37.5$ to the final compositional atomic percent ratios $\mathrm{Ca}: \mathrm{Ga}: \mathrm{Ge}=37.8: 25.2: 37$. The race of loss of $\mathrm{Ge}$ from the melt was subsequently found to be sensitive to temperature and oxygen partial pressure, with decreased evaporation at lower temperatures and higher oxygen partial pressures.

The second complication that occurred during crystal growth was solid state precipitation in the crystal. Although $\mathrm{Ca}_{3} \mathrm{Ga}_{2} \mathrm{Ge}_{3} \mathrm{O}_{12}$ is normally clear the single crystals often contained white opaque regions. These regions were shown by way topography to consist of decorated linear defects. The decoration consisted of $100 \mu \mathrm{m}$ long 1 inear arrays of small globules. In addition clear seed crystals appeared to become opaque during the growth process. The defects were not characterized Eurther.

In an effort to minimize these complications several adjustments were made to the growth procedure. 9 Varying the crystal rotation rate between 27 and 55 RPM did not eliminate the linear defects. The evaporation of Ge could be limited but not elininated by restricting the ventilation of the space over the melt surface and by admitting a high oxygen partial pressure. However, placing an alumina housing over the hole in the crucible 1 id to create an isothermal environment for the growing crystal and to permit pressure build-up of the volatile gases did not improve crystal quality significantly. Likewise varying oxygen partial pressure Erom 0 to 760 corx in the normal 760 torr imposed nitrogen atmosphere above the melt did not improve crystal 
quality significanty. The investigators ${ }^{9}$ note that local overheating of the melt should be avoided to grow high qualicy crystals since $\mathrm{GeO}_{2}$ evaporation is so sensitive to temperature.

B. Transmission Electron Microscopy Specimen Preparation

A boule of $\mathrm{Ca}_{3} \mathrm{Ga}_{2} \mathrm{Ge}_{3} \mathrm{O}_{12}$ approximately $4 \mathrm{~cm}$ long and $2 \mathrm{~cm}$ in diameter was supplied by Philips Laboratories, Findhoven, The Netherlands. TEM specimens were prepared as follows:

1) Slices approximately 10 mils thick were cut perpendicular to the boule axis. The boule axis was parallel to $<001>$.

2) Discs 3 m in diameter were cut ultrasonically.

3) The discs were mounted on a glass slide with a low melting point $\left(-150^{\circ} \mathrm{C}\right)$ resin and mechanically thinned on $600 \mathrm{grit}$ emexy paper until the specimen was 1.0 mil thick (as measured using a micrometer).

4) The thinned specimen was dissolved off of the slide using echyl alcohol and carefully transferred to a 3 m diameter copper grid which was lightly coated with epoxy glue.

5) After allowing the epoxy to dry, the specimen was ion milled until electron rransparent regions were detected. Typical ion thinning parameters were:
a. $5 \mathrm{kV}$ accelerating potential for argon ion guns
b. $25 \mu$ ion bean current
c. $25^{\circ}$ angle of impingenent
d. as long as 80 hours of thinning time were required, with a typical duretion of 55 hours. 
Atcempts to expedice ion thinning of specimens resulted in samples unsuitable for TEM studies. Specimens were thinned for about 16 hours with an acceleracing voltage of $9 \mathrm{kV}$, ion beam current of $40 \mu \mathrm{A}$ and an angle of impingement of $25^{\circ}$. After this the voltage was reduced to $6 \mathrm{ky}$, the beam current to $30 \mu \mathrm{A}$, and the thinning angle to $15^{\circ}$ in an attempt to produce a central hole in the specimen having thin edges and free of ion induced damage. After about 5 more hours of thinning under these conditions the specimens were found to have no central hole but many small perforations with edges too thick to permit adequate electron transmission.

Before observation in the electron microscope, a layer of carbon approximately $40 \mathrm{~A}$ thick was vacum evaporated onto one surface of each specimen to remedy problems associated with electrostatic charge build-up in the insulating crystals while in the electron microscope. 


\section{Electron Diffraction and Imaging}

This section deals with the practical problems encountered and procedures used in the course of the transmission electron microscopy experimentacion. High voltage transmission electron microscopy (HVEM) was performed using the Lawrence Berkeley Laboratory Hitachi HU $650 \mathrm{kV}$ instrument equipped with a high angle $\left(+20^{\circ}\right)$ double tilt goniometer stage. HVEM permits the use of thicker ion-milled CGGG specimens because of the increased penetration at high voltages and facilitates high ordex bright field imaging for increased resolution of lattice defects. 17 High ordex bright field imaging is especially applicable at high voltages due to the Flatness of the Ewald sphere permitting excitation of high order reflections through careful specimen tilting. The image is formed using the transmitced beam while a high order diffracted beam $n \vec{g}$ is excited (usually $n=4$ in this study). Since background intensities are high under such conditions focussing is easy and exposure times are short, minimizing loss of image resolution due to mechanical or other instrument instabilities. Image detail and resolution, such as those of dislocations ${ }^{18}$, are improved because of the long extinction distance $\left(\xi_{g}\right)$ associated with high order

reflections and the dependence of image width on $\left(1 / \mathrm{s} \xi_{\mathrm{g}}\right){ }_{0}^{19}$ The deviation parameter s can be altered by tilting the specimen and monitored by observing the Kikuchi line near the operating high order reflection. Increased resolution of lactice defects such as dislocations can occur (depending on $\overrightarrow{\vec{g}}$, specimen oxientation, and electron wavelength $\lambda$ ) if $s$ is slightly positive, i.e. the Kikuchi line lies outside of the 
operating reflection as is seen in the inset diffraction pattern from the high order bright field micrograph of Fig. 6A.

Weak-beam dark field imaging is another specialized technique permitting increased resolution of lattice defects and easier image interpretation. ${ }^{20}$ Weak-beam images are obtained by forming a dark field inage using a first order Bragg reflection $\vec{g}$ when the crystal is oriented such that the high order refection $\overrightarrow{n g}(n>1)$ is operating. This effectively increases the deviation parameter $s$ of the imaged reflection and results in increased resolution since diffraction contrast axises only at the core of defects such as dislocations. 21 Ease of specimen tilting to excite high order reflections is again facilitated at high voltages making weak-beam imaging a valuable rechnique of HVEM.

The advantages offered by weak-beam and high-order bright field imaging are qualitatively illustrated in Fig. 6. A specimen of $\mathrm{Ca}_{3} \mathrm{Ga}_{2} \mathrm{Ge}_{3} \mathrm{O}_{12}$ is imaged at $650 \mathrm{kV}$ in the sequence of micrographs and evidence of dislocation lines around chemical microsegregates can be seen. The improved resolution of dislocation images is apparent in the high-order bright field and weak-beam dark field images of Figs. 6A and C respectively as compared to the conventional bright field inage of Fig. 6B. These techniques giving increased resolution of latrice defects were utilized often in this study of defects in CGGG and circumvented some of the dynamical contrast phenomena which hindered interpretation of images for other investigators in studies of different garnet systems. 22,23

Diffraction pacterns obtained from undefective CGGG matrix regions often displayed "extra" spots as can be seen in ig.7. A symetrical 
[100] cubic diffraction pattern is indexed in $A$, and it is clear that $<200>$ type reflections are present. This is confirmed by calibration of the microscope camera constant ( $\lambda \mathrm{L}$ ma) using a ring patcern obtained fron a polycrystalline gold standard foil and shows correspondence with $<200>$ type incerplanar spacing. Upon tilting the specimen away from the $1001 \mid$ pole to obtain either of the mutually perpendicular highorder two beam cases shom in $B$ or $C$ of Fig. 7 , some spots having $<200>$ periodicity are seen to disappear. Scructure factor rules for the garnet crystal structure ${ }^{24}$ show $n<200>$ where $n=0$ dd integers to be unallowed reflections. The appearance of these unallowed systematic reflections in symetric diffraction patterns from a variety of major poles could be accounced for by double diffraction. Dark field imaging of the unallowed spots gave no evidence that they arose from second phases, microstructural defects, or anything other than double diffraction. Extra double diffraction spots are not normally observed in pacterns from perfect crystals. However in the diamond cubic and heragonal close packed structures extra spots can be observed. ${ }^{25}$ This is the case also for the complex b.c.c. type structure of CGGG. In the example of $\mathrm{Ig}$. $7 \mathrm{~A}$, the <200>type spots occur by double diffraction among $\langle 420\rangle,\langle 422\rangle$, and $\langle 400\rangle$ reflections. These reflections are the three grrongest reflections as listed on the ASTM card ${ }^{10}$ for $\mathrm{Ca}_{3} \mathrm{Ga}_{2}\left(\mathrm{GeO}_{4}\right)_{3}$ having $I / I$, values of 100,65 , and 65 respectively. Thus extra spots in the symetric difraction pattern occur at positions which are the vectoral sums of strong allowed diffxaction vectors, i.e., if $\vec{g}_{1} \ldots 420>$ and $\overrightarrow{\mathrm{g}}_{2}=\langle$ b00 $>$ then double diffraction and the resultant sum $\vec{g}_{1}+\vec{g}_{2}=\vec{g}_{3}=020>$ gives $x$ ise to a spot in the normally unallowed 
<020> position. Upon tilting to the systematic row or two beam cases such as $F i g .7 B$ and $C$ the $<200>$ type reflections disappear since at least one of the contributing allowed reflections is no longer strongly diffracting. While double diffraction is a viable explanation for the observed phenomena it should be noted that extra $<200>$ type spots in [001] rype patcerns from silicon ${ }^{26}$ have been actributed to dynamical effects among successive Laue layers in the diffraction pattern reciprocal space. It is important to distinguish between such anamolous appearances of reflections and other causes such as diffraction from second phase particles. 


\section{V-A. Microsegregate Defects/Results}

Chemical inhomogeneities in the CGGG samples could be identified by their dark contrast against the lighter matrix in TEM micrographs. Two closely-spaced defects of this type are imaged in bright field at $650 \mathrm{kt}$ in Fig. 8. The interace between the defective region and the matrix is sharp and shows no evidence of dislocations despite being obscured by the encireling periodic lobes of intensity. In the sequence of $F i g$. $8 A-D$ the operating diffraction $\vec{g}$ vector rotates clockwise in the plane and the lobes of contrast distort so as to tend to maintain a line of no contrast perpendicular to $\vec{g}$. The lobes are also seen to overlap in the region between the two chemical defects where the periodic incensity variations are incerrupted. The projected shape of each inhomogeneity, as is best seen in Fig. 8B, is approximately elliptical with najor and minor axes of lengths $0.25 \mu$ and $0.20 \mu$ respectively. The defects are situated such that their major axes are nearly colinear. Bright field stereonicrographs indicate these defects to consist of an oblate ellipsoidal volume which can be generated by rotating the projected elliptical area about the major asis of the ellipse.

Lobed "buteerfy" contrast of this sort is generally attributed to a coherenc lattice imperfection which strains the matrix. ${ }^{27}$ strain contrast can be described in terns of a displacement vector $\vec{R}$ due to the local alcering of the matrix by the imperfection. No contrast arises there $\vec{g} \cdot \vec{R}=0$, giving a line of no contrast perpendicular to g for a spherically symetrical strain field. Qualitative treatment of the strain concrast from the defects of Fig. 8 confirm these to 
not be strictly spherical but rather ellipsoidal. The periodic intensity variation is absent in the region between the defects where the strain fields overlap and the displacement vector $\vec{R}_{1}$ due to one defect is about equal in magnitude but opposite in direction to the displacement vector $\vec{R}_{2}$ of the other closely-situated defect.

The region marked $\mathrm{K}$ in $\mathrm{Fig} .8 \mathrm{~A}$ is representative of microstructural features commonly encountered in the CGGG samples. Such defects were often in close physical proximity to the chemical inhomogeneities although they could also be found in otherwise undefective CGGG matrix material. Contrast behavior from such features was quite sensitive to difracting conditions and specimen orientation. Sometines Exinged contrast was observed in such defects although the fringe intensity variation and orientation relative to $\overrightarrow{\vec{g}}$ showed no consistent behavior. It was not possible to make these defects go out of contrast by tilting the specimen. This contrast behavior led to the belief that these were not dislocations or internal boundaries like twins or stacking faults. Combined with the observation that cracks radiating from the ion milled hole in the specimen showed similar contrast, it is safe to assume that such features are cracks in the material. The origin of such cracks is not known and was not studied, so they will not be mentioned further except to be identified as cracks also designated $\mathrm{k}$ in subsequent micrographs.

Another morphology of the chemical inhomogeneities was also commonly observed. The bright field micrograph of Fig. 9 shows such defects to appear as dark regions about $0.4 \mu$ in size in a lighter matrix, but a light circular patch about $0.15 \mu$ in diameter is seen central 
to the interior of the inhomogeneity. The interface between the matrix and defective region is again quite well defined. However no evidence of strain contrast is apparent in the vicinity of the defect.

Bright field stereomicroscopy of such defective regions shows the dark area to have a shape similar to a cylinder but with an irregular non-circular cross section. Steromicrographs establish the light patch to be nearly spherical in shape and situated close to the geometrical centex of the dark region. The matrix/defect interface is found to be curved and not crystallographic or facetted in nature. Figure 10 shows another such chemical inhomogeniety with the light circular patch in a thin area of an [001] oriented specimen as imaged in bright field. Evidence of dislocations can be seen in the regions designated d. Rigorous analysis of such dislocations is reserved for a subsequent section. The dislocations are located at each corner of the defect and are in contact with the matrix/defect interface. This interface appears facetted with the projected interface image lying almost parallel co<110>type directions. The circular patch is slighty displaced from the center of the defective region and is seen to consist of two concentric annuli of different intensity denoted $s$ and m. Inside the region bounded by ring s the transmitted electron beam passes undiscurbed, i.e. as through a hole. This concentric ring contrast is consistent with the notion that this feature is spherical in shape provided it is also hollow. Thus the inner annus $s$ is the projection of where the sphere is cut by the surface of the specimen and the outer annulus $m$ is where the sphere reaches its widest equatorial dimension within the middle of the specimen. The region within ring 
$s$ is most intense since the transmitced beam passes without encountering any naterial, while a slightly darker intensity gradient is detected between rings s and due to the varying thickness of material encountered by the electron beam in that region.

The two morphologies of chemical inhomogeneities just described were observed often in the course of this study and the rollowing differences were generally maincained: (1) inhomogeneities with spheres near their centers showed evidence of dislocations in close proximity yet never internal to the chemical defects, (2) inhomogeneities with spheres and dislocations do not show pronounced strain contrast, and (3) inhomogeneities without spheres (and without dislocations) do display the lobed strain constrast which changes predictably with variation of difracting conditions.

Despite these stated differences, certain similarities were consistently maintained by the two types of inhomogeneities. A general observation was that the dark, chemieally inhomogeneous region was always darker than the matrix in both $B F$ and $D F$ and independent of the diffracting conditions. This is verified by the BF/DF pair of Fig. 11 which shows that inhomogeneities (with or without the central sphere) appear dark against a lighter matrix. The spherical entity is also seen to appear as a circular parch lighter in contrast than the matrix in both $B F$ and $D F$.

Another feature common to the two rypes of inhomogeneities is that electron diffraction patterns taken from either type of defective region are identical to patterns taken from the undefective CGGG matrix. No extra spots or streaks re resolved in the patterns, nor are any 
spots deleted. While extra spots were observed in symetric diffxaction patterns obtained near major crystallographic poles, they could be accounted for by considering double diffraction phenomena as explained earliex. In addition, these extra spors in symetric selected area diffraction pattens arose irregardless of whether the area selected was undefective matrix material or whether a chenical inhomogeneity was within the SAD aperture. Furthermore, rigorous dark field imaging of diffracted beans in several samples and various orientations failed to reveal any diffraction spots artributable solely to a chemical inhomogeneity. Based on this electron diffraction evidence the defective regions are believed to maintain the garnet crystal structure.

The density and distribution of these inhomogeneities are difficult to generalize. Electron microscope observations revealed that the distribution of defects varied from region to region even within a single TrM sample. The defects tend to be localized, i.e., it was common to observe highly defective areas approximately lou in diameter containing on the order of 100 such defects adjacent to areas $10 \mu$ in diameter which were completely undefective. Examples of defect densities spanning the entire range just established could also be Found depending on the particular specimen selected and the area viewed. Despite the finding that undefective garnet matrix regions having dimensions as large as $50 H$ could be traversed in the electron microscope, the chemical defects observed in the CGGG samples can be qualitarively described as prolific.

In regions of relatively high defect density the chemically inhomogeneous regions could be found to be distributed in a variety 
of ways. Often the inhomogeneities appeared to be randonly distributed in the matrix, but ordered distributions were also common. Figures $12 A$ and $B$ show adjacent regions in [001] oriented CGGG specimen as imaged in BF. Emamples of inhomogeneities with and without a central sphere can be found and the distribution of defects in the matrix is obviously not random. Linear arrays are observed aligned parallel. to the mutually perpendicular $<100>$ crystallographic directions in the plane of the page. Although arrays aligned parallel to $<110>$ were also observed in some areas, alignment parallel to $<100>$ was much more comon. In the micrograph of Fig. $12 \mathrm{~A}$ a chemical defect with a central sphere is seen at $S$. This defect is bounded by semicircular arcs having a line of no contrast perpendicular to the operating g. This is suggestive of the contrast obtained from prismatic edge dislocation loops having Burgers vectors normal to the plane of the loop and a line of no contrast where $\overrightarrow{\vec{b}} \cdot \vec{b} \times \vec{u}=0$ (u) being a vector parallel to the dislocation line dixection). Such dislocation loops are discussed in detail in a later section but it should be noted that the observation of such a loop is consistent with the generalization that defects having a central sphere often have dislocations in close proximity to them but no strain contrast is displayed.

Lobed strain contrast associated with the inhomogeneities not having a central sphere can also be seen in Fig. 12 . It is most apparent in the region L of Fig. $12 B$ where evidence of strain field overlap is visible around the linear array of closely spaced defects. In the region $L$ as well as elsewhere a very faint yet detectable line of light contrast is seen connecting the defects, running parallel 
to the $\langle 100\rangle$ axis of defect alignment and through the centers of the defects. Detailed rilting of the specimen was not performed to determine it the extra intensity is due to diffraction contrast from the overlapping strain fields.

The observation of linear arrays of defects in TEM samples aided in identifying defects by optical microscopy in 10 mil thick slices cut from the sample boule perpendicular to its growth axis. Using transmitted light and magnifications of $600 x$, white opaque regions were locally distributed randonly as well as in linear arrays. Since individual regions were of an appropriate size (i.e. $0.5 \mathrm{~mm}$ at $600 \mathrm{x}$ ) they are believed to be the same chemical inhomogeneities observed in the electron microscope. The distribution of these defects as established by optical microscopy confirms the TEM findings that defects tend to be prolific but localized; they occur in random or linear arrangements, and localized regions of undefective matrix are also comon. Furthermore, it was qualitatively established that defect densities and configurations did not vary significantly in regions close to the boule growth/rotation axis as compared with regions near the periphery of the boule. Defect densities as a function of length along the boule axis were not established by optical nor TEM methods. V-B. Microsegregate Defects/Discussion

The two types of chemical inhomogeneities which have been observed (with and without the central sphere) are believed to be the same type of defect except at different stages of development. This is supported by the similarities in size, shape, and contrast displayed 
by each as well as the observation of both types of defects interdispersed in linear arrays.

Both types of defects appear as dark regions in the lighter matrix in both $B F$ and DF, suggesting that the defective regions exhibit greater absorption of electrons in boch transmitced and diffracted beams than does the CGGG matrix material. Since the electron diffraction evidence suggests that the defects have the same garnet structure as the matrix, this contrast could arise from a compositional difference between the matrig and the defect. Such a compositional variation tends to alter the intensity of beams passing through the material in two ways as follows: (1) by introducing different atomic scattering factors $f(\theta)$ into the structure factor $\left(\sigma_{g}\right)$ sumation, and (2) by variations in unit cell volume $V_{c}$. Thus compositional changes can cause intensity variations since the amplitude $\sigma_{g}$ of an electron wave $i^{s}$ proportional to $\mathrm{F}_{\mathrm{g}} / \mathrm{V} \mathrm{c}$ and the intensity is proportional $\theta_{\mathrm{g}} \emptyset_{\mathrm{g}}^{*}$. Since absorption seems significant in interpreting the contrast from the defects and since electron absorption cross sections generally increase with increasing atomic number, it is tempting to think that the dark contrast arises from replacing some of the elements in the CGGG matrix with elements of higher atonic number in the defective regions. On this premise it is suspected that the dark region is rich in $G a$ or Ge since for typical reflections used $(\sin \theta / \lambda=0.6-0.7)$ the atomic scattering factors for these elements are about 1.5 times as large as that for $\mathrm{Ca}^{27}$ However this qualitative notion is subject to shortcomings because it fails to quantitatively account for the actual latrice positions which such substitutional atoms occupy in the unit cell and how electron wave 
amplitudes and intensities will be affected by constructive and destructive interference phenomena when the atomic scattering factors and atomic positions are incorporated into the structure factor calculation.

Therefore the observed contrast could result from differences in garnetstructure lattice site occupation between the defect and matrix although the compositional nature of this difference cannot be deduced from contrast behavior alone. Compositional variations between matrix and defective garnet regions can also account for the observed strain contrast due to lattice mismatch since garnet lattice parameters are sensitive to substitution of elements.

The light contrast displayed in both BF and DF by the spherical feature central to some of the chemical inhomogeneities can be explained in a reverse manner, that is the sphere is composed of material which allows greater electron transmittance. The spherical morphology is indicative of a hydrostatic pressure such as that exerted by entrapped gas. All of the above observations can be explained if the sphere is assumed to be filled wich oxygen gas under pressure. The spherical morphology and hollowess follow as a direct consequence while the light contrast is explained in terms of the low atomic number and hence small absorption cxoss section of oxygen permitting greater transaittance of the electron beams. The source of this accumulated oxygen could be the constituent oxides in the crystal growth melt itself or the atmosphere of 760 torr oxygen pressure imposed above the melt during growth.

A specimen was observed at $650 \mathrm{kV}$ in a Hitachi Hu650 electron microscope equipped wich a heating stage. No dimensional changes 
were detected in the chemical inhomogeneities or internal spheres which were imaged before and after heating for 4 hours at $650^{\circ} \mathrm{C}$. Heating to higher temperatures is expected to cause enlargement of the oxygen filled sphere since oxygen pressure could then overcome the surface energy tending to reduce the surface area (and hence the volume) of the sphere. Temperatures near the CGGG melting point of $1380^{\circ} \mathrm{C}$ are suggested.

The nature of the matrix/defect interface has been deduced from the observation of strain and dislocations. The interface is always very distinct and it is considered to be coherent for inhomogeneities showing strain contrast while coherency is partially lost when dislocations are generated in the matrix near the interface and the evidence of strain is greatly reduced. The sharpness of the interface implies a very strong short range force maintaining it and a chemical force is suspected. Attempts to further characterize the interface using direct latrice imaging have not succeded due to complications from excessive contamination of the specimen in the electron microscope as well as insufficiently thin ion-rilled specimens.

Observation of strain fields around some of the defects can be correlated with the linear aligment of the chemical defects. Similar practical observations of aligned precipitate particles have been made in other systems ${ }^{28}$ with the comment that the transformation of one particle tends to cause the transformation of neighboring particles along certain crystallograhic directions. An elastic interaction between the chemical inhomogeneities is likely since they are separated by distances comparable to their dimeter and evidence of overlapping 
strain fields has in fact been observed (Figs. 8 and 12). Nabarro 29 cites unpublished work by Crum which showed that there was no interaction between centers of dilatation in isotropic crystals and that the dilatational stress field of one coherent particle does not affect the energy needed to form another similar particle nearby. This indicates that since interaction between strain centers is observed, the cubic garnet structure of CGGG is not isotropic.

Cahn ${ }^{30}$ has considered the interaction of dilatational strains in elastically anisotropic cubic crystals and the pertinence of strain energies to nucleation in solids. He gave a mathematical treatment showing that the strain energy associated with a particular point in a small transformed volume depends on the strain at that point as well as strains at other points in the volume and the strains due to 211 other particles in the system. Using this approach Cahn concluded that, given a premexisting precipitate particle, it is energetically favorable to nucleate a second particle in an elastically soft direction. Furthermore, the relative values of the material elastic constants determine the soft direction and if $2 C_{44}-C_{11}+C_{12}>0$ then aligment of particles will occur along $<100>$ (while if $2 \mathrm{C}_{44}-\mathrm{C}_{11}+\mathrm{C}_{12}<0$ then alignment parallel to $\langle 11\rangle$ is favored). The observation of linear arrays parallel co<100> directions in CGGG indicates the fomer condition co be true of the elastic constants for CGGG. Cahn ${ }^{30}$ suggests that $<100>$ directions are 21 so favored in most metallic systems, and the elastic constants of several garnets indicate that <lo0> alignwent would be favored alchough the elastic constants for $\mathrm{Ca}_{3} \mathrm{Ga}_{2} \mathrm{Ge}_{3} \mathrm{O}_{12}$ are not avalable. 31 
Cahn's treatment was extended to consider a particle with two neighbors of equal sige and at equal distances, but with one neighbor in a hard direction and the other in soft direction. Due to free energy considerations the particle in the soft direction will have a lower solubility in the matrix than the particle in the hard dixection and hence is more stable. As a result, even if particles initially nucleated and grew at random then this enhanced stability would dictate the development of an ordered array, i.e. a simple cubic patcern if $<100>$ directions are soft. Thus it is possible that the observed alignment of the chemical inhomogeneities is a post-nucleation phenomena whose driving force is derived from strain energy. This is not in agreement with the studies of microsegregation in CGGG reported by Mishra and Thomas ${ }^{32}$ who suggest such Iinear defect arrays to form on existing dislocation lines which act as pipes for easy segregate diffusion.

Linear arrays of interspersed defects with and without the central sphere were common as was mentioned previously and can be seen in Fig. $12 A$ and $B$. Since alignment of defects is atributed to the scrain field interactions between neighboring inhomogeneities and inhomogeneities showing pronounced strain effects genexally did not have a central sphere, it is reasonable to conclude that alignment of defects occurs due to strain interactions among defects not having a central sphere. However, defects having a central sphere and not exhibiting significant strain are also observed in the linear arrays indicating that formation of a sphere is a post-alignment process. This is not to say that a defect must occur in a linear array in order 
to form aphere because many instances of defects with spheres (as well as their associated dislocations) isolated in otherwise undefective matris have been encountered. Instead it is suggested only that the strained chemically inhomogeneous regions not having a sphere are a preliminary stage in the formation of the defects which have developed the central oxygen-filled sphere. A qualitative model for sequential defect transformation stages and their relation to crystal growth melt conditions and processing parameters is included in the 1.ast section of this paper. 
VI-A. Chemical Analysis/Results

Chemical micro-analysis of the inhomogeneous regions was performed using scanning transmission electron microscopy (STEM) with energy dispersive analysis of $x^{-x a y s}$ (EDAX). Limited access to chemical microanalysis equipment was gained by traveling to Rockwell Science Center in Thousand Oaks, California to use their Philips 400 transmission electron microscope equipped with a Philips scanning electron inaging unit. A lithium-drifted silicon detector manufactured by Nuclear Semi-Conductor was used for counting $x-r$ ays, and the resulting numerical data was processed using a Tracor/Northern NS 880 analyzer. The identical CGGG samples used in HVEM studies were used for this analysis. Chemical inhomogeneities both with and without the central sphere were investigated using a $200 \AA$ dianeter probe of $120 \mathrm{keV}$ electrons. The experimencal procedure and results are shown schematically in Fig. 13. The electron probe was first placed in an otherwise undefective CGGG matxix region about one micron away from a chemical defect and spectral counts were recorded for a counting period of 200 seconds. Chemical information was recorded as digitized spectra (counts of a particular energy versus energy) on computer printout as well as polaroid pictures of a CRT graphic display (see Fig. 14). This process was repeated with the 200 probe central to the dark chemical defect. In the case of chemical defects having a central sphere the probe was placed on the raddle of the dark annular region which encircles the sphere (see Fig. 13). Altogether four different chemical defects were investigated, two with and two without the central sphere, and their corresponding matrix spectra were also each 
recorded. Spectra were also recorded with the probe placed central to the light area of the sphere for both of the defects displaying a sphere.

Qualitative analysis of the $k_{\alpha}$ peaks of the resulting spectra showed several consistent results as follows: (1) All matrix and defect regions showed evidence of only five elements, those being $\mathrm{Ca}, \mathrm{Ga}, \mathrm{Ge}$, and smaller amounts of $\mathrm{Fe}$ and $\mathrm{Cu}$. No oxygen was detected since the STEM/EDAX unit will not resolve species having atomic number less than eleven. (2) Al1 defect spectra showed the relative heights of the $\mathrm{K}_{\infty}$ peaks for $\mathrm{Ga}$ and $\mathrm{Ge}$ to be reversed with respect to their corresponding matrix spectra, $i . e .$, the Ge peak was more intense than the $G$ peak in matrix spectra whereas the Ga peak was more intense than the Ge peak in spectra Erom the chemical defects. (3) Spectra obtained with the probe placed central to the spheres showed inconsistent and uninterpretable behavior.

Quantitative analysis using the method of Goldstein, et a1. ${ }^{33}$ was done by manually replotting the digitized spectra so that the areas under the $\mathrm{K}_{\mathrm{o}}$ peaks could be more accuracely calculated for each of the matrig and defect regions. The data generated is tabulated in the appendix where the analytical procedure is 21 so outlined. Three assumptions were made in the analysis: (1) the area under a peak for a given elenent is directly proportional to the number of atoms of that element present in the volume of material from which chemical information is being received, (2) the matrix material is stoichiomerric $\mathrm{Ca}_{3} \mathrm{Ga}_{2} \mathrm{Ge}_{3} \mathrm{O}_{12}$, and (3) the defective region is a garnet of composition $\mathrm{Ca}_{\mathrm{x}} \mathrm{Ga}_{y} \mathrm{Ge}_{2} \mathrm{O}_{12}$ where carion sites are conserved with 
64 cations per unit cell or 8 cations per formula unit. Therefore, by taking the appropriate ratios of areas under the peaks from matrix regions and comparing with the stoichiometric cation atomic fraction ratios it was then possible to deduce a nominal composition of the defective regions from the ratios of the area under their STEM/EDAX specra peaks.

The calculated composition $\mathrm{Ca}_{\mathrm{x}} \mathrm{Ga}_{\mathrm{y}} \mathrm{Ge}_{z} \mathrm{O}_{12}$ of the chemically defective regions is given in Table 1 of the appendix. The results show good agreement although those labelled STEM 6 (from a chemical defect with a sphere) are slightly anomalous. The nominal composition of chemical defects having spheres (STEM 3 and 6 ) is very comparable to that of defects not having a sphere (STEM 9 and 11). All defects show the composition $\mathrm{Ca}_{\mathrm{y}} \mathrm{Ga}_{\mathrm{y}} \mathrm{Ge}_{\mathrm{z}} \mathrm{O}_{12}$ to be rich in gallium and depleted in germanium relative to the $\mathrm{Ca}_{3} \mathrm{Ca}_{2} \mathrm{Ge}_{3} \mathrm{O}_{12}$ matrix since $y$ is greater than two and $z$ is less than three in each case. The calcium content of both matrix and defect seems similar since z approximately equals three. By simply taking an axithmetic average of the $x, y$, and $z$ parametexs an average nominal composition of the chemically inhomogeneous material is found to be $\mathrm{Ca}_{3} .12^{\mathrm{Ga}} 3.39^{\mathrm{Ge}} 1.48^{\mathrm{O}} 12^{\circ}$

VI-B. Chemical Analysis/Discussion

The results just presented are subject to limitations inherent in the experimental and analytical methods used. The average nominal

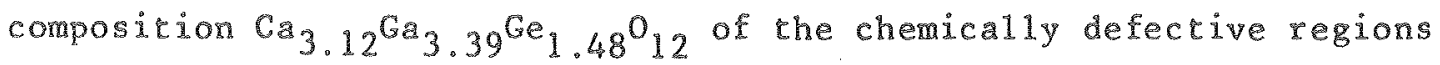
is a result which must be interpreted accordingly. It is likely that there are compositional variations or gradients even within the chemically defective regions and a nominal composition is of questionable 
significance. It is equally likely that detailed high resolution chemical microanalysis would reveal differences in composition between the inhomogeneities with a sphere and those without one. Consequently an average composition is of limited value. In addition it was found that during the chenical analysis expeximent the electron probe was subject to small amounts of dxift relative to the specimen. Thus there results some uncertainty in the area from which chenical information was obtained despite the efforts of the experimenters to minimize this variable. There arises another uncertainty in the composition of the inhomogeneities since they lie central to the depth of the sample. Chemical information is thus likely to have also been obtained from colums of matrix material between the specimen surface and the defect interface and not uniquely from the chemical defect irself. Most of these shortcomings could be minimized through careful operation of the STEM/EDAX apparatus and selectively choosing the areas to be analyzed.

The assumptions used in the quantitative analysis are also subject to limitations. It is generally accepted that the area under a spectral peak for a cercain element is proportional to the amount of that element present, and chis assumption is not subject to much debate. The second assumption was that the matrix naterial was stoichiometric $\mathrm{Ca}_{3} \mathrm{Ga}_{2} \mathrm{Ge}_{3} \mathrm{O}_{12}$ This was necessary since the STEM/EDAX instruments had not previously been calibrated with samples of known $\mathrm{C2}, \mathrm{Ga}$, and/or Ge concentration for a more accurace determination of the matrix composition. In fact the instruments mere to be calibrated for $\mathrm{Ca}, \mathrm{Ga}$, and Ge for future 
reference on the basis of the matrix spectra experimentally generated, with the assumption that the spectra came from stoichiometric $\mathrm{Ca}_{3} \mathrm{Ga}_{2} \mathrm{Ge}_{3} \mathrm{O}_{12}{ }^{\circ}$

The third assumption is subject to debate since it is conceivable that the chemically defective garnet regions may not have sixty four cations per unit cell (or eight per formula unit). This assumption that $x+y+z=8$ was necessary since the ratios of areas under the peaks of spectra from the inhomogeneities did not alone yield unique solutions for the parameters $x, y$, and $z$ of the defect composition $\mathrm{Ca}_{8 \mathrm{~g}} \mathrm{Ga}_{\mathrm{y}} \mathrm{Ge}_{z} \mathrm{O}_{12}{ }^{\circ}$ A similar assumption based on garnet electroneutrality (i.e., $2 x+3 y+4 z=24$ ) could have been employed. This alternative was not used since local variations from electroneucrality are suspected to be involved in the formation of the chemical inhomogeneities. Likewise no experimental evidence has been found of cation vacancies in the inhomogeneous regions, so the assumption of conservation of cation sire occupancy was adopted.

The chemical information obtained shows several significant features despite the rallibility of the analysis. Chemical inhomogeneities with and without spheres are undoubtedly related phenomena as indicated by the similarity in their elemental composition. In addition, both result from interactions mong chemical species intrinsic to the CGGG system, i.e. Ca, Ga, and Ge. Athough traces of Fe and Cu were consistently detected, they were attributed to the iron pole pieces in the scanning transmission electron microscope and the $3 \mathrm{~mm}$ copper grid on which the specimen was mounted. This was verified by the presence of only $F e$ and $C u$ peaks in background spectra 
recorded while the electron beam was directed through the center of an ion-milled hole in the specimen without impinging on the specimen itself. Consequently the chemical defects seem to result from small scale segregation of gallium in the garnet structure. These microsegregates, as they may be referred to henceforth, are depleted in germanium, enriched in gallium, and equivalent in calcium content relative to the $\mathrm{Ca}_{3} \mathrm{Ga}_{2} \mathrm{Ge}_{3} \mathrm{O}_{12}$ matrix.

A garnet having composition $\mathrm{Ca}_{3.12^{\mathrm{Ga}}} 3.39^{\mathrm{Ge}} 1.48_{12}^{\mathrm{O}}$ would have a larger lartice parameter than $\mathrm{Ca}_{3} \mathrm{Ga}_{2} \mathrm{Ge}_{3} \mathrm{O}_{12}$ since garnets obey Vegard's rule $e^{5}$ and the ionic radius of $\mathrm{Ga}^{3+}$ is larger than that of $\mathrm{Ge}^{4+}$ by about $0.009 \AA$. The lactice parameter for such a garnet would be on the order of $12.26 \AA$.

To maintain electroneurralicy a garnet of composition $\mathrm{Ca}_{3} .12$ $\mathrm{Ga}_{3} .39^{\mathrm{Ge}} 1.48^{\mathrm{O}} \mathrm{v}$ would need $8 \times(3.12 \times 2+3.39 \times 3+1.48 \times 4)=176$ units of negative charge per unit cell. This could be supplied by only eighry nine $0^{2-}$ ions, and there would be surplus oxygen in a region of such a garnet phase if the normal ninety six oxygens per garnet unit cell are available. This surplus oxygen could be involved in the formation of the oxygen filled sphere, with the accumulation of oxygen motivated by electrostatic imbalance as the crystal cools.

The dark appearance of the microsegregates in both BF and DF is not explicable in terms of the chemieal analysis alone. Since gallium has a lowex atomic scattering factor than does germanium, the gallium-rich microsegregate region might be expected to appear lighter than the matrix and this is not observed. This indicates that the absorption processes within the segregated regions are dependent 
on structural as well as chemical factors and detailed theoretical analysis of constructive and destructive interference phenomena would be needed to correlate the multitude of possible cation distributions among the available sites with the observed dark contrast.

The deficiency of germanium displayed by the microsegregates indicates that defect formation is related to the evaporative loss of germanium reported to have occurred during crystal growth. It is possible that as germanium evaporates, for whatever reason, gallium segregates to occupy their normal octahedral sites as well as the tetrahedral sites vacated by the lost germanium. In fact, gallium is know to preferentially occupy the tetrahedral sites in other garnet systems 5 and it is likely that such sites in CGGG would easily accomodate the $\mathrm{Ga}^{3+}$ ions since oxygen packing around tetrahedral sites is less dense than the packing around octahedral sites. Calcium, being the largest cation, cannot fit into octahedral nor tetrahedral interstices and it is not surprising that calcim occupancy of dodecahedral sites is maintained in the microsegregated regions.

Little insight has been gained about the chenical nature of the spheres central to some microsegregates. Since oxgyen is resolvable by very few chemical analysis techniques, the nature of the sphere nay have to be deduced in the future rather than established directly. If evaporation of germanium occurs during crystal growth even with an imposed atmosphere of 760 torr oxgyen partial pressure, then segregation of Ge may also be expected around a sphere if it is filled with oxygen gas under pressure. Consequently a germanium concentration gradient may be found to exist in the region of inhomogeneity between the maxtrix/ 
microsegregate interface and the sphere incerface in a microsegregate with an oxygen filled sphere. The higher oxygen pressure of the sphere could attract germanium much as the atmosphere above the growth melt does and germaniw concentration near the surface of the sphere may be higher than elsewhere within the microsegregated volume. This rype of gradient would have to be established by further high resolution chemical micro-analytical techniques.

Chemical analysis of the defects was originally attempted using 2 different scaning electron microscope equipped with EDAX. The resolution of that instrument was inadequate to distinquish the composition of the microsegregates fron that of the matxix. However, in an effort to expose more of the ricrosegregated volume in the SEM specimens, they were placed in $250^{\circ} \mathrm{C}$ phosphoric acid for one hour in order to etch away the matrix material. Stereo analysis of the SEM nicrographs showed linear arrays of pits about $0.5 \mu$ in size. These pits are of about the same size and distribution as was found for the microsegregates using TEM techniques. Thus it seens that the microsegregate regions were preferentially attacked by the acid. This is significant because it shows that chemical thinning technques using hot $\mathrm{BH}_{3} \mathrm{PO}_{4}$ (as is used for other garnets) are not viable methods for preparing TuM samples of CGGG for microsegregate analysis. 


\section{VII-A. Dislocations/Results}

Dislocations in the CGGG samples tended to be in close physical proximity to the microsegregate defects. They were found to always be in the matrix material at or near the defect interface but never internal to the defects. Although isolated matrix dislocation loops such as those reporced by Mishra and Thomas ${ }^{32}$ were also observed, it can be generaliged that the density of dislocations not associated with the chemical defects was low. Dislocation lines and loops were seen but other types such as regular or sheared dislocation helices were

not encountered as have been reported in other garnet systems. 34

The dislocation lines and loops assumed many different geometries, with one example already seen in Fig. 6. Despite this diversity three major rypes of dislocations were identified. They have been classified as Types 1, 2, and 3 for brevity of later reference to these dislocation configurations, and a description of each type follows. (1) Dislocations specified to be Type 1 can be seen in the regions labelled d of Fig. 10. These are dislocation lines which lie primarily in the matrix material but are in contact with the defect/matrix interface. Such dislocations are usually associated with isolated microsegregates which: (a) are not in a linear array, (b) have developed a central sphere, and (c) tend to have a Eaceted or crystallographic (rather than curved) sort of incerface. The dislocations are inclined to the specimen surfaces and situated at each corner of the defect/matrix interface. Dynamical contrast phenomena have hindered characterization of Type 1 dislocations, however it is certain that these are dislocation lines and not segments of dislocation loops which encixcle the 
inhomogeneous region. This is true since the dislocations could be made to go out of contrast individually whereas segments of a dislocation loop retain the same Burgers vector and would be expected to disappear simultaneously. No evidence of dissociation into partial dislocations was found. Specimen tilting for $\vec{g} \cdot \vec{b}$ analysis gives agreement with the previously published results of Mishra and Thomas ${ }^{32}$ that the Burgers vectors of such Type 1 dislocations lie paralle 1 to $<112>$ crystallographic directions. (2) Type 2 is the designation given co dislocations of the sort seen in region $d$ of Fig. $15 \mathrm{~A}$ and $\mathrm{B}$. The dislocations are out of contrast in $C$. These are dislocation lines lying entirely in the matrix region near an isolated microsegregate containing a sphere. Evidence of interactions between the dislocation and the chemically defective region can be seen. The inhomogeneity has a predominantly curved interface although the section of interface nearest the dislocations is seen to have a flat, linear projection oriented almost parallel to the dislocation line direction. The sphere is seen to be displaced from the center of the inhomogeneity so as to be near this flattened interface and the dislocations. The mottled appearance of the background in the micrographs of Fig. 15 is attributed to the carbon film deposited on the specimen surface. Type 2 dislocations are deduced to be of mixed screw and edge character having Burgers vectors parallel to <113> directions which are neither strictly parallel nor perpendicular to the $210>$ dislocation line directions. They are assumed to be perfect since evidence of dislocation dissociation in weak-beam dark field or high order bright field micrographs has not been found. (3) The third comnon configuration of dislocations 
is illustrated in Fig. 16. A microsegregate with a sphere is imaged in bright field under three different diffracting conditions. Two dislocation loops about $0.5 \mu$ in diameter are seen to encircle the defective region in Fig. 16A. The loops are viewed nearly edge-on in a direction parallel to [001]. These loops are mutually perpendicular to each other and extend beyond the defective region at its equator into the matrix material where evidence of strain contrast is faint yet detectable. In the micrographs of $\mathrm{Figs}, 16 \mathrm{~B}$ and $\mathrm{C}$ the specimen was tilted to make each of these Type 3 loops go out of contrast in turn. Each loop is seen to disappear when the operating diffraction vector $\vec{g}$ lies parallel to the edge-on projection line of the loop. Trace and $\overrightarrow{\mathrm{g}} \cdot \overrightarrow{\mathrm{b}}$ analyses reveal such dislocation loops to be of edge rype and no evidence of dissociation of the loops into partial dislocations is seen. The Burgers vector for each loop is of magnitude $a_{0}<100>$ and is directed perpendicular to the $\{100\}$ plane on which the loop lies.

Another example of Type 3 loops encixcling a chemical defect with a sphere is seen in the weak-beam dark field micrograph of Fig. 17. The view is parallel to [120] and one loop on (001) is seen edge-on while evidence of multiple dislocation images on the mutually perpendicular (010) plane can be seen $2 t d$. No evidence of intensity rringes can be seen between the multiple images. The edge-on loop was made to go out of contrast in diffracting conditions consistent with Burgers vector of $a_{0}<001>$ perpendicular to the plane of that 10op. Kowever during specimen tilting the contrast from the loop or loops giving rise to the double dislocation image did not show 
disappearances as anticipated for the expected Burgers vector(s) parallel to [010]. Although the Burgers vectors were not definitively established they are not parallel to $\langle 010\rangle$. Dynamical contrast phenomena can account for the white blotches of intensity seen in the micrograph of Fig. 17.

The incerstitial or vacancy nature of the Type 3 dislocation loops was not ascertained since inside/outside contrast was negligible upon tilting to and from known crystallographic poles.

VII-B. Dislocations/Discussion

The fact that dislocations interact with the microsegregate defects is readily apparent. Chemical forces and strain energy associated with the microsegregates are suspected driving forces for these interactions. The particular defect configuration observed depends not only on the complex chemistry and crystallography of the CGGG system but also on the material processing parameters. The interplay between strain and chemical forces is indicated by the absence of $a_{0} / 2<111>$ Burgers vectors. While this is the shortest perfect b.c.c. lattice translation vector it has been theoretically shown ${ }^{15}$ to imply strong electrostatic contributions to the defect energy. This accounts for the alternative Burgers vectors experimentally observed to be parallel to $<001>$, $\langle 112\rangle$, and $\langle 113\rangle$ 。

Dislocations near the microsegregates act to relieve strain due to lattice mismatch between the garnet structures of the matrix and the microsegregates. This is suggested by the fact that all dislocations were found to lie primarily in the matrix and is apparent from the reduced matrix' strain contrast associated with dislocated 
micxosegregates. It is possible that the various types of dislocations were always in the matrix material and were later attracted to the region of the growing chemical inhomogeneity. Under this circumstance it might be expected that matrix dislocations would be seen in the vicinity of the strained chemical defects (not having central spheres) and these are seldom observed experimentally. Instead it is more likely that dislocations are generated by the growing microsegregates. Size of the chemical defect alone ig not a critical factor governing dislocation generation since strained chemical defects have been observed in the same area as dimensionally smaller dislocated microsegregates (which also had the internal oxygen filled sphere). Strain energy per unit volume and segregate concentxation, which are not completely independent parameters, are more likely to govern dislocation formation. A correlation between dislocation generation and sphere formation has been alluded to but it is not a straightforward one. While it is true that microsegregates having Type 1, 2, or 3 dislocations were always seen to have the oxygen filled sphere in their interiox, it was not uncormon to see microsegregates with spheres which showed no evidence of such dislocations. Consequently it seems that sphere fomation and dislocation generation are mutually influential processes. Qualitative evidence of such an influence is seen by the sphere of Hig. 15 being displaced from the center of the microsegregate volume coward the dislocation line in the matrix.

To ascertain the nature of the interaction between a microsegregate and a Type 1 or Type 2 dislocation line would require atomistic deternination of the permutations in lattice positions and consequent 
electrostatic and strain energies associated with their respective Burgers vectors of $a_{0}\langle 112\rangle$ and $a_{0}\langle 113\rangle$. This was not accomplished but will be necessary in the fucure if the role of Type 1 and Type 2 dislocations in microsegregation phenomena in CGGG is to be fully appreciated. Type 1 dislocations are probably generated near the corners of the facetred microsegregates due to stress concentrations in those regions.

The Type 3 dislocation loops of igs. 16 and 17 are always found to encircle the microsegregates and lie in the matrix material several hundred angstrons away from the interface. This suggests that the dislocation accomodates matrix strain and that while the dislocation may have been generaced at the interface it has grown into the matrix by climb. These Type 3 loops lying on $\{001\}$ planes are seen faceon in region $\mathrm{S}$ in the plane of the $\{001\}$ oriented foil of Fig. 12A and give rise to the split-ring inge typical of edge dislocation loops. The line of no contrast perpendicular to the operating $\vec{g}$ occurs since the invisibility criterion $\overrightarrow{\mathrm{g}} \cdot \overrightarrow{\mathrm{b}} \times \overrightarrow{\mathrm{u}}=0$ is satisfied at these locations $\vec{u}=$ a unit vector tangent to the dislocacion loop at any point on the 1oop).

The double dislocation image of region d of Fig. 17 can be intergreted as follows: (1) a double image of a single dislocation line, (2) one inage each of two different concentric loops, or (3) two partial dislocations separated by a region of stacking fault. The result that the $100 \mathrm{p}$ does not have the usual a $<001>$ Burgers vector gives support to alternatives (2) and (3). Alternative Burgers vectors might be expected to make two concentric perfect loops stable 
whereas Burgers vectors of a $2001>$ may be preferred for the single Type 3 loop configuration. Likewise two concentric partial dislocation loops may be stable due to energy reductions from dissociation of a perfect loop. The perfect loop could have any of a number of Burgers vectors, but for a Type 31 loop with $\vec{b}=a_{0}<001>$ several dissociations are possible which conserve octahedral cation positions. 15 Future verifications of the perfect or partial nature of these multiple loops and their Burgers vectors will give considerable insight into the elastic and electrostatic energies which govern crystal defects in CGGG.

The possibility that the double dislocation image arises from a single dislocation must not be overlooked. Mishra and Thomas 32 have shown through calculations of theoretical image profiles that double images of a single undissociated dislocation can still arise despice the increased resolution obtained from weak-beam dark field imaging.

Mechanisms for dislocation loop generation around chenical inhomogeneities prepe propod by J.W. Mathews ${ }^{35}$ as a result of his work on gadolinium gallium garnet. Similar mechanisms have been proposed in metallic systems as wel1. ${ }^{36}$ A mechanism proposed by Macthews to account for the formation of single loops around inclusions requires the formation of a small prismatic loop outside of the inclusion (see Fig. 18A). The loop grows by the addition of point defects as show in Fig. 18B. This is a mistit dislocation accomodating lattice mismatch between the inclusion and the natrix. However, if the concentration of point defects is very high in the vicinity of the loops, then the growth of the loop may continue via climb until the configuration 
of Fig. $18 \mathrm{C}$ is reached. The loop remains near the equatorial plane of the inclusion and is stable with respect to glide because of the interaction between the strain fields of the loop and of the inelusion.

Once a single large loop has grown, another concentric loop may form and grow by repeating the same process. This can account for the observation of two concentric and apparenty coplanar dislocation loops in Fig. 17. Likewise, the two images could be from a single dislocation formed by the above process which dissociated into partials. Stacking fault fringes might not be seen between the partials if the splitting distance is small and the extinction distance is large (since Exinge spacing $t_{\alpha} \xi_{g}$ ).

A similar mechanism proposed by Mathews to account for multiple loop generation begins with the formation of a small prismatic dislocation loop immediately outside of the inclusion (see "a" of Fig. 19). This loop has its plane normal to both the surface of the inclusion and the Burgers vector of the 1oop. Growth occurs by absorption of interstitials or emission of vacancies (for an interstitial loop) until a crescent-shaped configuration is achieved (like "b" in Fig. 19). The point defects are activated by the elastic strain energy due to the inclusion. The strained nature of the segregated regions of Iig. 8 supports the viability of this mechanisn for the CGGG system.

Continued growth permits portions of the loop to meet on the opposice side of the inclusion where they annihilate leaving a pair of concentric loops $c_{1}$ and $c_{2}$. Two main forces act on loop $c_{2}$ and the relative magnitudes of these forces determines whether a loop like $c_{2}$ is glissile. One force is due to the inclusion and tends 
to increase any movement of the loop away from the equatorial plane of the inclusion. The other force is due to the misfit dislocation $c_{1}$ and tends to return the outer loop to the equatorial plane. Still another force may need to be considered in garnets such as CGGG. The Burgers vectors of perfect dislocations could favor loops $c_{1}$ and $c_{2}$ having partial Burgers vectors with the loops connected by an area of stacking fault. These stacking faults could hinder motion of the loops away from the equatorial plane. The above discussion could also account for the observation of double dislocation images in $\mathrm{F}$. 17. Both of Matthew' theories were developed for the case of an isotropic material. While the 1 inear arrays of microsegregates indicate anisotropy in CGGG it is possible that the anisotropy will not preclude the operation of either of the above loop formation mechanisms, but rather it may dictate the types and configurations of dislocations which are found. 
VIII-A. Coherent Precipitates/Results

Another distinct defect configuration commonly encountered can be seen in Fig. 20. These look like small loops about $0.1 \mu$ in size and are not physically associated with established microsegregate defects as are dislocation Types 1, 2 and 3 . Contrast from the interior of such loops is not clear but it sometimes appears that a fringe or some other feature may be present in some. The matrix material has a pocked appearance suggestive of the presence of point defects. The density and distribution of the pocked appearance and the small loops was unaffected by long exposure (several hours) to an intensely focussed beam of $650 \mathrm{kV}$ electrons. Several general observations were made concerning the distribution of the small loop-like features. (1) They were not distributed homogeneously throughout the bulk of the samples. Where the density of the larger microsegregates was very high, the density of these small loops and point defects was low or zero. (2) Where the small loops were observed they were seen in great numbers and found to always show evidence of pocked background.

Contrast from these loop-like features was very sensitive to diffracting conditions such as the particular operating reflection excited and the deviation parameter s. Image quality was improved using $s$ slightly positive as determined in the diffraction mode by the Rikuchi line outside of the operating diffxaction spot. Stereonicroscopy showed these loops to be distributed fairly uniformly throughout the thickness of the specimen whereas the pocked appearance seemed to be localized near the surfaces. Loops seemingly near the specimen 
surface often displayed anomalously wide images (see Fig. 21) which could not be improved with high-resolution BF or DF imaging techniques.

Selected area diffraction patterns from the areas of the sample where the sma11 loops were profilic did not contain extra spots, streaks, or other anonalies not resolved in S.A.D. patterns obtained from undefective CGGG matrix.

Despite meticulous tilting of the specimen the small loops could not be made to go out of contrast completely. Loops viewed face-on commonly show a line of no contrast through the loop in a direction perpendicular to the operating diffraction vector $\vec{g}$ (Fig. 22). Stereographic analysis of faceron and edge-on loops in the [001] oriented specimen of Fig. 22 showed the loops to be lying on $\{001\}$ type planes, however similar analysis of other loops indicated that alternative habit planes are also likely. Systematic tilting of the specimen failed to reveal a consistent scheme of inside/outside contrast for determining intrinsic or extrinsic nature of the loops.

Several of the small loops can be seen in very close proximity to each other in region $C$ of $F i g .20$. The identity of the individual loops has been obscured in the region where their images overlap. Stereomicroscopy of these loops indicates that coalescence of the disc-like loops into a three-dimensional feature has occurred as opposed to merely appearing to overlap in the projected image. Evidence of such coalescence of small loops was not uncommon.

The pocked appearance of the matrix around the small 1 loops displays several contrast effects. The small "points" are observed to reverse contrast across an extinction contour in Fig. 21. 
In addition, the BE/DF pair of Fig. 20 shows the points to have a black-white lobe contrast. The direction $\vec{q}$ of white to black contrast is seen aligned both parallel and antiparallel to the operating diffraction vector $\vec{g}$, depending on the particular point under consideration. Furthemore, examples of the line of black to white contrast being either identical or reversed for a particular point in a BF/DF pair can be found. These types of contrast behavior are usually atroibuted to point defect clustexs in the specimen ${ }^{37}$, with contrast being governed by the diffracting conditions, the incerstitial or vacancy nature of the defects, and theix depth distribution in the foil.

\section{VIII-B. Coherent Precipitates/Discussion}

Contrast from the small loop-like features indicates that they may be either dislocation loops or else coherent or partially coherent precipitates. Distinguishing between these various possible defects is sometimes difficult on the basis of contrast phenomena alone. other workers ${ }^{38}$ have been able to differentiate these features by mechanically and thermally crearing their specinens and determining whether the observed microstructural changes and properties correlace more closely with dislocation or precipitate behavior. Such thermomechanical treatments were not performed on the CGGG samples. However, qualitatively it is easier to explain formation of three-dimensional defects in terms of coalescence of precipitaces racher than dislocations. Also, prismatic loops might be expected to show very weak or zero contrast in some diffracting conditions where $\overrightarrow{\mathrm{g}} \cdot \overrightarrow{\mathrm{b}}=0$, and no reflection was found which caused virtul disappearance of any of these features. Additional evidence will be presented later to support the notion 
that these are small disc shaped precipitates. Consequenty it is assumed that they may be coherent or partially coherent precipicates since these can each theoretically give rise to contrast similar to that observed experimentally. Partially coherent precipitates comonly show interfacial mistit dislocations but evidence of such dislocations was not found. Wurthermore it is reasoned that the precipitates are likely to be coherent since partially coherent precipitates often have a structure different from the matrix and give rise to extra spots in the diffraction pattern which are unique to the precipitate. Such extra spots were never isolated using DF methods and therefore the loops are attributed to $0.1 \mu$ diameter disc-shaped coherent precipitates.

A coherent precipitate can give rise to contrast very similar to a prismatic dislocation loop of partial "Burgers vector" $\vec{b}$ provided that the strains in the plane of the disc are very small and the major displacements of magnitude $\left|\vec{b}_{p}\right|$ occur in directions perpendicular to the plane of the disc. When such a disc is viewed face-on, the experimentally observed line of no contrast will be seen at points where $\vec{g} \cdot \vec{b} \times \vec{p}=0$, where $\vec{u}$ is a unit vector tangent to the edge of the disc at any point. Since $\vec{b}_{p}$ is normal to the plane of the disc and $\vec{u}$ lies in it, $\vec{g} \cdot \vec{b} x \vec{p}=0$ only when $\vec{u}$ lies parallel to $\vec{g}$ and a line of no contrast is seen perpendicular to $\vec{g}$ (where $\vec{g}$ and $\vec{u}$ are parallel). Such a line of no contrast pexpendicular to $\overrightarrow{\mathrm{g}}$ can be seen in $\overline{\mathrm{F}} \mathrm{ig} .22$. Wide images such as those of Fig. 21 have been observed from coherent precipitates in other systems as well. ${ }^{27}$ These are generally attributed to surface relasation effects of strain fields from precipitates lying within half an extinction distance of the foil surface. 
The thickness of the discs as established by measurement of the minimum image width of edge-on precipitates tended to be on the ordex of a few hundred angstroms. Dynamical contrast from the precipitates has hindered a more specific determination. It is doubtful that the precipitates are substantially thinner than this since streaking in the diffraction patterns along dixections normal to the projected images of edge-on discs would be expected but was not observed experimental1y.

Several factors indicate that the small loop-like precipitates are related to the larger microsegregate defects. Specirmen often contained both types of defects except in different areas which were usually separated by regions of undefective matrix. Both types of defects are observed in the bulk of the specimens suggesting that they are defects intrinsic to CGGG and not just specimen preparation artifacts. The $\{100\}$ habit plane of the small precipitates is reminiscent of the $\{100\}$ habit plane of the large edge dislocation loops surrounding the microsegregates with spheres. These precipitates could conceivably act as nucleation sites for the small climb loops of Mathews theory. 35 In addition, the observed collescence of several precipitates into a three dimensional mass indicates that the precipitates are a preliminary stage in the formation of the larger wicrosegregate defects. The three dimensional microsegregate morphology is obtained from the two dimensional disc-shaped precipitates since they are randomly distributed on the three mutually perpendicualar $\{100\}$ cube faces in the lattice. 
Chemical analysis was performed on the small precipitates in an effort to correlate their composition with that of the larger microsegregates. An analysis was conducted using STEM/EDAX by placing a $200 \AA$ dimeter probe of $120 \mathrm{keV}$ electrons cencral to a sacemon precipitate for a specific counting time and comparing the chemical information chus obtained with information gotten by the same methods from undefective CGGG matrix regions. Representative STEM/EDAX microanalysis spectra are shown in Fig. 23. Two major conclusions can be made as follows: (1) the precipitates show no evidence of elements extrinsic to the CGGG system (except Fe and Cu as explained previously), and (2) the composition of the precipitate shows negligible difference from that of the matrix. Consequently the precipitates are not due to inclusion of elements extrinsic to the CGGG sample, such as platinum from the crystal growth crucible.

It is likely that careful re-execution of this experiment will reveal the precipitates to be rich in gallium and depleted in germanium as would be expected for the coalescence model of microsegregate formation. The reasons for not verifying this in the original chemical analysis are three-fold: (1) The specimens used for the analysis were thick relative to the thickness of the small precipitates. Consequently substantial amounts of matrix material would be found in colums above and below the precipitates and the chemical information from this matrix material masks the information obtained from the precipitate. (2) The STEM instrument ws subject to instabilities which make the probe move relative to the target (or vice versa) by distances on the order of 500A. Thus there is anice probability that chemical information 
was being received from areas other than the original precipitate target area despite the best efforts of the experimenters to eliminate this source of error. (3) No high resolution chemical analysis apparatus was available ar U.C. Berkeley or Lawrence Berkeley Laboratory necessitating travel to Rockwell Science Center where access to such equipment was 1 imited in duration.

Stereomicroscopy has shown that the Eeatures giving rise to the pocked appearance of the matrix tend to be localized near each of the specimen surfaces. The various contrast phenomena observed, i.e., the line of black to white contrast, the identical or complementary nature of contrast in DF/BF pairs, and the reversal of contrast across an extinction contour, are consistent with contrast from point defects or clusters located near both surfaces of the specimen. If these point defects are not homogeneously distributed in the thickness of the specimen they are probably not defects intrinsic to CGGG. They are not likely to arise due to irxadiation in the electron microscope because their density would be expected co change while under the influence of the electron beam and they should be preferentially located near only the top surface of the foil where the electron beam impinges. Therefore they are expected to axise from two-sided ion milling of the samples which accounts for their distribution near both surfaces of the TEM specimens. The strain energy from the precipitates contributes to the process of cluster formation, making the area adjacent to such precipitates more susceptible to ion damage. Controlled ion thinning experiments could be done in the future to confirm the origin of such defects in CGGG. 
The possibility that these clusters are intrinsic to CGGG and exist in the bulk of the specimens camnot be precluded because of the thickness of the samples used. Ascertaining the depth distribution of these clusters was hindered by the mottled contrast expected from such clusters if they are located near the center of a specimen which is several extinction distances thick. Such clusters in the bulk could be involved in precipitate and microsegregate formation. The density of the clusters would then be expected to be low in the vicinity of large microsegregates (as is observed experimentally) since the clusters could be consumed during precipitate tormation and subsequent growth into larger microsegregates. 
IX. Mode1 for Microstructure Formation

The results and discussion presented suggest a model of microsegregate defect formation which entails nucleation and successive stages of growth of the chemically inhomogeneous regions. The multiplicity of observed defect configurations is too great to allow a general theory accounting for 211 of them. Consequenty this model is concerned only with the formation of a microsegregate defect having a central oxygen-filled sphere and encircled by a single undissociated Type 3 dislocation loop.

Defect formation comences with the random nucleation of the small loop-like precipitates show in Figs. 20-22. This occurs due to the preferential evaporative loss of Ge from the melt and results in local deviations from stoichionetry. Evaporation rate is sensitive to temperature ${ }^{9}$ (and oxygen partial pressure) and consequently thermal fluctuations in the melt solution can be responsible for localized germanium 1oss. As the germanim is lost through evaporation the small loop-like second garnet phase is formed by incorporating $\mathrm{Ga}^{3+}$ ions in sices of the growing crystal which would normally be occupied by $G e^{4+}$ ions.

Random nucleation continues due to loss of Ge from the melt and is followed by growth of the coherent loop-like precipitates which generate strain. As growth procedes the strain fields of neighboring particles start to overlap. Continued growth and interaction of over lapping strain fields then enhance coalescence of closely spaced individual precipitates into a larger chemical defect, as is indicated in region $\mathrm{C}$ of Fig. 20. The result of such coalescence of small 
loop-like precipitates is the larger strained chemically inhomogeneous regions (without the central sphere) as is seen in Figs.8, 11 and 12.

Coalescence of small precipitates into the larger, strained chemical defects continues until the population of the larger defects is great enough and their physical separation small enough to permit interaction of overlapping strain fields. Strain field interactions then motivate the alignment of the defects along crystallographically soft directions as dictated by the anisotropic elastic constants of the material. This notion of formation of 1 arger chemical defects from the coalesence of smaller loop-like precipitates and their subsequent alignment due to strain is consistent with the observations of Fig. 24. The bright field micrograph of an [001] oriented CGGG specimen shows the larger chemical inhomogeneities (some with evidence of the incernal sphere) aligned along [100] anidst a proponderance of the randomly distributed small loop-like precipitates. The region of material on either side of the linear array is depleted of the small precipitates. Such a denuded band around the larger defects verifies their fomation by coalesence of the small loops. ${ }^{28}$

Evidence supporting the succession of events up until this stage of defect fomation has been gained through HVEM. Mowever the material factors and chronological succession of events leading to further formation of the internal oxygen filled sphere and/or encircling dislocation loop has not been ascertained. It is probable that both processes occur upon cooling the crystal and proced as follows. Formation of a single dislocation loop could occur by the method of Matthews ${ }^{34}$ as shown in Fig. 18. This method requires formation of a small 
prismatic loop outside of the microsegregate. Such small prismatic loops have been observed to nucleate around small precipitates in other systems 36,39 and the small loop-like precipitates found in CGGG are suspected to act as nucleation sices. The loop is nucleared due to strain which is enhanced upon cooling and grows to successively larger diameters (Fig. $18 \mathrm{~B}$ and $\mathrm{C}$ ) by climb. Point defects (vacancies or interstitials) are absorbed or emitted by such a climb loop during growth until it reaches the equatorial position around the microsegregate where strain energy stabilizes it with respect to glide. The observed evidence of point defects in CGGG suggests that they arise due to ion milling and would consequently not be involved in loop formation. To verify the mechanism of loop formation in CGGG would require determining the interstitial or vacancy nature of the 100 as we11 as further characterization of the type, distribution, and origin of the point defects in CGGG. Size of the microsegregate defect does not seem to be the only critical factor in loop formation since relatively large $(0.6 \mu)$ as well as small $(0.2 \mu)$ microsegregates have been observed with and without encircling loops. Instead strain energy per unit volume is suspected as a critical factor since it is independent of size.

Generation of the mutually perpendicular Type 3 loops seen in Fig. 16A requires only that the individual loops nucleate around small precipitates lying on mutully perpendicular $\{100\}$ planes. Three mutually perpendicular loops on $\{100\}$ could conceivably encircle a microsegregate. This is not observed experimentally since one of the $\langle 100\rangle$ was coincident with the crystal pulling/rotation axis during 
growth of the CGGG boule. Thus during processing the $<100\rangle$ pulling direction was the zone axis for two equivalent $\{010\}$ (on which loops form) whereas the remaining $\{100\}$, whose nomal was the pulling axis, was different.

Formation of the oxygen-filled sphere is likely to accompany or follow relief of microsegregace strain since microsegregates with the central sphere seldon show pronounced strain contrast. The oxygen within the sphere can be supplied by the constituent garnet oxides of the melt or the imposed oxygen partial pressure of 760 torr above the melt solution. Since the size of the sphere is always observed to be proportional to the size of the microsegregate in which it is found (i.e., the diameter of the sphere is always about one third of the largest dimension of the microsegregate) the oxygen is probably supplied from the melt solution in the following manner. The evaporation of Ge permits accomodation of $\mathrm{Ga}^{3+}$ ions on tetrahedral sites of the growing crystal normally occupied by $\mathrm{Ge}^{4+}$ ions. This substitution results in a metastable phase at the growth temperature since the size of $\mathrm{Ge}^{4+}$ and $\mathrm{Ga}^{3+}$ ions do not vary greatly and hence small strains are involved. The metastable nature of these regions is indicated by the preferential etching of the microsegregates by hot acid. In the regions where $\mathrm{Ga}^{3+}$ has been substituted for $\mathrm{Ge}^{4+}$ there would be $a$ deficiency of positive charge. This can be remedied by supplying additional positive charges or eliminating some negative charge. Supplying positive charge is not feasible since this is most easily accomplished using the $\mathrm{Ge}^{4 t}$ ions which evaporate. Instead negative charges are deleted by the leaching out of $0^{2-}$ anions as the crystal 
cools. Since the matrix surrounding the nicrosegregate defect is stoichiometric $\mathrm{Ca}_{3} \mathrm{Ga}_{2} \mathrm{Ge}_{3} \mathrm{O}_{12}$ with the proper amount of oxygen, the $0^{2-}$ anions diffuse away from the matrix, i.e., coward the center of the microsegregate. Accumulation of these anions occurs to form the oxygen filled sphere whose shape is maintained by the pressure of the accumulated oxygen gas.

From this model it is suggested that defect formation could depend upon evaporative loss of Ge from the melt. Thermal conditions in the melt solution and growth atmosphere have been show to influence the rate of loss of $\mathrm{Ge}^{9}$ Consequently any effort to eliminate the microsegregace defects must address these factors. Specifically, germanium loss due to evaporation may be reduced by imposing a higher Geo partial pressure above the melt (instead of $\mathrm{O}_{2}$ ). The vapor pressure of $\mathrm{Ge}$ and $\mathrm{GeO}_{2}$ above their pure substances near the CGGG crystal growth temperature are on the order of $10^{-3}$ torr. However, Geo is a substantially more volatile component with vapor pressures exceeding 760 torr at $1000^{\circ} \mathrm{C} .40$ Thus germanium loss from the melt may be due to the volatility of Geo at high temperatures and imposing a Geo atmosphere above the melt may reduce the rate of germanium loss. The deposition of $\mathrm{GeO}_{2}$ on the crystal pulling shaft ${ }^{9}$ results by reaction of Geo gas upon cooling in the imposed oxygen acmosphere.

Thermal fluctuations within the melt would be minimized by controlling heat loss from the crucible as well as by insuring that unstable convective currents in the growth melt solution due to specimen wobble during rotation are eliminated. 


\section{Conclusions}

Several conclusions are apparent as a result of this electron microscopy study of calcium gallium germanium garnet.

(1) High voltage transmission electron microscopy and the techniques of weak-beam dark field and high order bright field imaging have permitted characterization of microsegregate defects and dislocations in CGGG.

(2) Lattice defects observed in CGGG are governed by the complex structure and chemistry of the garnet system. Such defects are of sufficient size and density that they could be detrimental in certain applications in which CGGG single crystals could be valuable, i.e., magnetic bubble memories.

(3) The formation of microsegregate defects is correlated with germanium loss from the crystal growth melt. Evaporative loss of germanium and subsequent microsegregation phenomena may be reduced by properly altering processing parameters. Imposing a higher GeO partial pressure above the melt and eliminating temperature fluctuations in the melt are suggested. 
Suggestions for Further Study

Additional work can be performed in the following areas to further this characterization of CGGG defects.

Ion thinning of TEM specimens must be done systematically to determine if the observed point defects are truly specinen preparation artifacts. Techniques for producing thin and uncontaminated CGGG samples suitable for lattice imaging should be developed.

HVEM studies of dislocations and possible dissociations should be pursued using weak-beam DF and high order BF imaging. Such studies could provide insight into the structural and electronic interactions associated with CGGG defects.

Lactice imaging studies of microsegregate/matrix interfaces can establish aspects of coherency, orientation, and possibly compositional Eluctuations.

Chemical analysis using high resolution STEM/EDAX to establish local chemical gradients and fluctuations within the bulk of the crystal as well as within individual microsegregate defects will provide further detail for the model of defect formation and suggest dependences on processing parameters.

Heat treatment near the CGG melting point in an appropriate atmosphere after crystal growth may prove beneficial in suppressing microsegregate and/or dislocation formation and growth. 


\section{Acknowledgements}

It is a privilege to acknowledge Professor Gareth Thonas for the guidance, encouragement, and opportunities he has provided throughout the duration of this study. I also wish to thank Professor Jack Washburn and Professor Dennis $w$. Hess for theix critical appraisals of the manuscript.

To Dr. Raja K. Mishra goes my most sincere appreciation for the invaluable time, effort, and Eriendship which he has invested to better my understanding of Materials Science and life in general.

Special thanks go to Dr. David R. Clarke for his collaboration on the chenical analysis portion of this paper as well as the use of the STEM/EDAX facility ar Rockwell Science Center in Thousand Oaks, California.

I am greatful for the assistance provided by the Lawrence Berkeley Laboratory staff during the various stages of this study. I wish to specifically thank Don Jurica and Carolyn Gosnel1 for their diligence in maintaining the TeM lab in working order, Gloria Pelatowski for her flawless reproduction of the line drawings, and all the Photo Lab staff for their punctuality in providing high quality work.

Heartelt gratitude is extended to my imediate tamily and my financee Marita Schad for the enduring support and encouragement which only they could provide.

Rinally I want to acknowledge J. B. M. Damen and J.A. Pistorius of Philips Laboratories for supplying the material samples used in this study. 
This research was performed under the auspices of the United States Department of Energy through the Matexials and Molecular Division of the Lawrence Berkeley Laboratory. 
Appendix: Chemical Analysis

Chemical analysis was performed at Rockwell Science Center, Thousand Oaks, California using a Philips electron microscope equipped with an x-ray microanalyzer. Chemical spectra from CGGG samples were obtained using a $200 \mathrm{~A}$ diameter probe of $120 k \mathrm{k}^{\mathrm{V}}$ electrons. The spectra thus obtained were recorded on computer printout as well as Polaroid photos of a CRT display. The area under a peak of the spectrum for a particular element is assumed to be proportional to the number of atoms of that element present in the area subjected to the electron beam.

Using the technique described above and the analysis which follows, four microsegregate defects (of about $0.4 \mu$ size) were investigated and found to have the Eollowing compositions.

\section{TABLE 1}

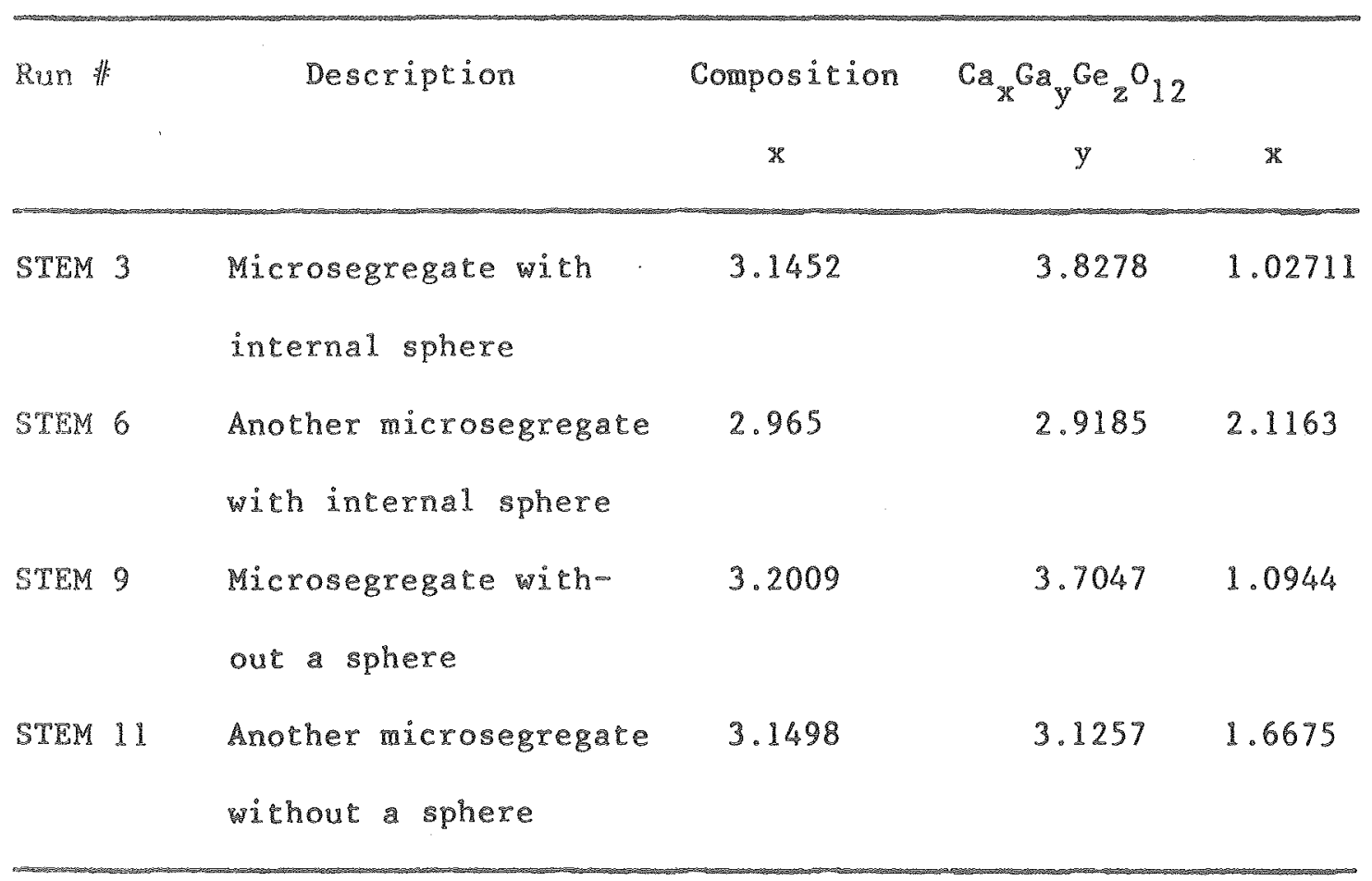


These results were generated from the following tabulated data which show measurements of spectral peak heights, widths at half maximum above background, and calculated areas under the peaks. STEM 1, 5, and 10 are from CGGG matrix regions located about $1 \mu$ from the chemical defects of STEM 3, 6, and 11 respectively.

TABLE 2. Marrix Analysis Data

\begin{tabular}{|c|c|c|c|c|}
\hline Run 非 & $\mathrm{K}_{\alpha}$ Peak for & Height & Width & Area \\
\hline \multirow[t]{3}{*}{ STEM 1} & $\mathrm{Ca}$ & 2790 & 0.152 & 212.04 \\
\hline & $\mathrm{Ga}$ & 1351 & 0.196 & 132.35 \\
\hline & $\mathrm{Ge}$ & 1840 & 0.208 & 191.36 \\
\hline \multirow[t]{3}{*}{ STEM 5} & $\mathrm{Ca}$ & 748 & 0.146 & 54.6 \\
\hline & $\mathrm{Ga}$ & 360.5 & 0.188 & 33.89 \\
\hline & $\mathrm{Ge}$ & 469 & 0.194 & 45.493 \\
\hline \multirow[t]{3}{*}{ STEM 10} & $\mathrm{Ca}$ & 1047.5 & 0.143 & 74.9 \\
\hline & $\mathrm{Ga}$ & 552.5 & 0.179 & 49.45 \\
\hline & $\mathrm{Ge}$ & 759 & 0.189 & 71.72 \\
\hline
\end{tabular}


TABLE 3. Microsegregate Analysis Data

\begin{tabular}{|c|c|c|c|c|}
\hline Run 非 & $K_{0}$ Peak for & Height & width & Area \\
\hline \multirow[t]{3}{*}{ STEM 3} & $\mathrm{Ca}$ & 1712.5 & 0.144 & 123.3 \\
\hline & $\mathrm{Ga}$ & 1479 & 0.190 & 140.5 \\
\hline & $\mathrm{Ge}$ & 395 & 0.184 & 36.34 \\
\hline \multirow[t]{3}{*}{ STEM 6} & $\mathrm{Ca}$ & 309 & 0.135 & 20.86 \\
\hline & $\mathrm{Ga}$ & 202 & 0.199 & 20.099 \\
\hline & $\mathrm{Ge}$ & 146 & 0.184 & 13.432 \\
\hline \multirow[t]{3}{*}{ STEM 9} & $\mathrm{Ca}$ & 1824.5 & 0.146 & 133.19 \\
\hline & $\mathrm{Ga}$ & 1562 & 0.188 & 146.83 \\
\hline & $\mathrm{Ge}$ & 418.5 & 0.195 & 40.8 \\
\hline \multirow[t]{3}{*}{ STEM 11} & $\mathrm{Ca}$ & 1599 & 0.153 & 122.32 \\
\hline & $\mathrm{Ga}$ & 1226.5 & 0.196 & 120.2 \\
\hline & $\mathrm{Ge}$ & 675 & 0.190 & 64.125 \\
\hline
\end{tabular}

The following is an illustrative calculation of the composition of the microsegregate of STEM 3. To determine this composition required a calibration of the microscope using the matrix spectra of STEM 1. Since the matrix is assumed to be stoichiometxic $\mathrm{Ca}_{3} \mathrm{Ga}_{2} \mathrm{Ge}_{3} \mathrm{O}_{12}$, then the ratio of atomic fractions of $\mathrm{Ca}$ to $\mathrm{Ga}$ in the matrix area of STEM 1 is $3 / 2$, whereas the ratio of Ge to Ga is also $3 / 2$ while the $\mathrm{Ca} / \mathrm{Ge}$ ratio is $3 / 3=1$. However the ratios of areas under the $\mathrm{Ca}$, 
Ge, and Ge peaks of STEM 1 shows

$$
\begin{aligned}
& (\text { Ca area } / \text { Ge area })=212.04 / 132.35=1.6021 \\
& (\text { Ge area } / \text { Ga area })=101.36 / 132.35=1.4459 \\
& (\text { Ca area } / \text { Ge area })=212.04 / 191.36=1.1081 .
\end{aligned}
$$

Therefore the actual ratio of areas under the peaks is related to the atomic Eraction ratios by

$$
\begin{array}{ll}
\mathrm{Ca} / \mathrm{Ge}=3 / 2=\mathrm{K}_{1}(1.6021) & \mathrm{K}_{1}=0.9363 \\
\mathrm{Ge} / \mathrm{Ga}=3 / 2=\mathrm{K}_{2}(1.4459) & \mathrm{K}_{2}=1.0374 \\
\mathrm{Ca} / \mathrm{Ge}=3 / 3=\mathrm{K}_{3}(1.1081) & \mathrm{K}_{3}=0.90245
\end{array}
$$

where $k_{1}, K_{2}$, and $k_{3}$ are lumped constants which account for such factors as the variability of the STEM/EDAX detector in identifying elements of different atomic number. These constants effectively calibrate the microscope detector since using this information the composition $\mathrm{Ca}_{\mathrm{x}} \mathrm{Ga}_{\mathrm{y}} \mathrm{Ge}_{\mathrm{z}} \mathrm{O}_{12}$ of the microsegregated region of STEM 3 can be deduced. Therefore the ratios of areas under the STEM 3 peaks are:

$$
\begin{aligned}
& \mathrm{Ca} / \mathrm{Ga}=\mathrm{x} / \mathrm{y}=\mathrm{K}_{1}(123.2 / 140.5)=\mathrm{K}_{1}(0.87758)=0.9363(0.87758)=0.821652 \\
& \mathrm{Ge} / \mathrm{Ga}=\mathrm{z} / \mathrm{y}=\mathrm{K}_{2}(36.34 / 140.5)=\mathrm{K}_{2}(0.25865)=1.0374(0.25865)=0.26833 \\
& \mathrm{Ca} / \mathrm{Ge}=\mathrm{K} / \mathrm{z}=\mathrm{K}_{3}(123.2 / 36.34)=\mathrm{K}_{3}(3.39296)=0.90245(3.39296)=3.06198 .
\end{aligned}
$$

However these ratios of the composition parameters $x, y$, and $z$ alone do not yield a single convergent solution to the composition. Therefore an assumption must be made and it is assumed that cation sites in the defective garnet regions are conserved so that $x+y+z=8$. Since

$$
x=0.821652 y
$$


and

$$
z=0.26833 y \text { then by solving } x+y+z=8
$$

it is seen that

$$
\begin{aligned}
& y=3.8278 \\
& z=1.02711
\end{aligned}
$$

and

$$
x=3.1452
$$

These are che values of the composition parameters found in Table 1 For STEM 3. The values for STEM 6, 9, and 11 are found by sinilar methods. A nominal composicion for general microsegregated volume was calculated by taking an arithmetic average of the $x, y$, and z para-

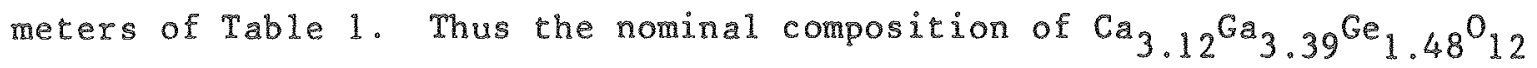
were derived. 


\section{ReFerences}

1. T. H. O'De11, Magnetic Bubbles, John Wiley and Sons, New York, 1974.

2. A. Hobeck, P.I. Bonyhard, and J. E. Geusic, Proc. IEEE, 63, 非8, 1975, 1176-1206。

3. P. Chaudhari, et al, Physics of Thin Films, 1977, 263-297.

4. E. A. Giess, et. 21., Mat. Res. Bu11., 8, 1973, 1061-1066.

5. J. E. Davies, and E. A. Giess, J. of Mat. Sci., 10, 1975, $2156-2170$

6. W. L. Roth, From The Chenistry of Extended Defects in NonMetallic Solids, North-Holland Publishing Co., London, 455-485.

7. M. J. Stowel1, from Epitaxial Growth, Part B, Academic Press, New York, $1975,382-489$.

8. J. W. Mathews, et. 1., Phys. Stat. Sol (a), 19, 1973, 671.

9. J. P. M. Damen, et al., Mat. Res. Bu11., 12, 1977, 73-78.

10. ASTM X-Ray Powder File Index Card $11-23$.

11. K. I. Standley, Oxide Magnetic Materials, 2nd Ed., Oxford University Press, 1972, 30-36.

12. R. W. G. Wykoff, Crystal Structures, Vol. 3 Interscience Publishers, Inc., New York, 1960, Chapt. XII, 3-5.

13. S. Geller and M. A. Gilleo, Phys. Chem. Sol., 3, 30, 1956.

14. J. Friede1, Dislocations, Rergamon Press, 1964, 149-160.

15. J. Rabier, B. Veyssiere, and J.Grilhe, Phys. Stat. Sol., (a), 35, 1976,259 .

16. J. P. M. Damen, and J.A. Pistorius, Private communication to Dr。R. K. Mishra, May, 1977. 
17. G. Thoms, Proc, 4th Int, Conf, on KVEM, Toulouse, France, 1975, p. 247.

18. R. Osiecki, and G. Thomas, $29 \mathrm{th}$ Ann, Proc. EMSA, Boston, Mass., 1971.

19. G. Thomas and M Goringe, Transmission Electron Microscopy of Materials, to be published, J. Wiley and Sons, New York, 1979.

20. D. J. H. Cockayne, J. De Physique, C7, 35, 非12, December, 1974, p. 141 .

21. J.W. Edington, Practical Electron Microscopy in Material Science, Monograph 非, MacMillan, 1975.

22. J. Rabiex, H. Garem, and P.Veyssiere, J.App1. Rhys., 47, 非11, $4755-58$.

23. B. Mardinan, et. a1., Phi1. Mag., 27, 1973, 777.

24. International Tables for X-Ray Crystallography, Vol. 1, The Kynoch Press, Birmingham, England, 1952, pgs. 55, 345-346, 524-525.

25. J.W. Edington, Practical Electron Microscopy in Material Science, Monograph 非, MacMiliian, 1975, 59-62.

26. K. N. Tu and A. Howie, Phi1. Mag, B, 37. 非1, 1978, 73-81.

27. P. B. Hirsch, et. A1., Electron Microscopy of Thin Crystals, Butterworths, $1965,96-97,328-332$.

28. H. F. Merrick, and R. B. Nicholson, 5th Int. Cong. For Electron Micros., Vol. 1, K8, Academic Press, N. Y., 1962.

29. F.R. N. Nabarro, Proc. Phys, Soc, 52, 1940, 90; Proc. Roy. Soc., A175, 1940, 519.

30. J. W. Cahn, From The Mechanisms of Phase Transforrations in Crystalline Solids, Inst. of Metals, London, 1969, 1. 
31. Landolt-Bonstein Tables, New Series, Group III, Vol. 1, p 10, 31, and Vol. 2, p 6-7, Springer-Verlay, New York, 1966.

32. R. R. Mishre and G. Thomas, J.App1. Phys., 49, 非3, March 1978, $1876-1878$

33. J.I. Goldstein, J. L. Costley, G. W. Lorimer, and S.J.B. Reed, Scanning Electron Microscopy/1977, 1, 315-24.

34. J.W. Matthews, et.21., Khys.Stat. Sol. (2), 28, 1975, 95-105.

35. J.W. Matchews, Phys. Stat, So1., (2), 15, 1973, 607.

36. A. Eikum and G. Thomas, Acta. Met., 12, May 19c4, $537-545$.

37. M. Wilkens, Exon Modern Diffraction and Imaging Techniques in Material Science, Ed. S. Anelinckx, et. a1., North-Holland Pub1ishing Co., 1970, 233-256.

38. D. Kull and I. J. Mogford, Phil. Mag, 6, 1961, 535.

39. X. H. Westmacot, et. 1., Phi1. Mag., I, 1962, 1585.

40. Davydov, V. I., Germanium, Gordon and Breach Science Publishers, New York, 1966, p. 144-187. 
Figure Captions

Fig. 1. A) Garnet dodecahedral or c sites have the least dense packing of oxygen and are occupied by the largest cations, $\mathrm{Ca}^{2+}$ in CGGG. An orygen ion is located at each of the eight vertices of the dodecahedron with the cation central to its interior. There are three c sites per garnet formula unir.

B) Octahedral carion sices are surrounded by six closely packed oxygen ions and axe usually occupied by the smallest cations. There are two octahedra (or "a") sites per chemical formula unir.

c) Tetrahedral coordination of oxygen provides d eation sites of size intermediate between the $a$ and $c$ sites in garnets. There are three such sites per formula unit and in CGGG these sites must be at least partially occupied by the snallest $\left(\mathrm{Ge}^{4 t}\right)$ ions.

Fig. 2. The cations are arranged in one octant of the unit cell such that the oxygen polyhedra share edges and vertices. Each oxygen is shared by one retrahedral, one octahedral, and two dodecahedral sites (one of which has been eliminated in the figure for clarity).

Fig. 3. A) Each octant of the unit cell contains one garnet formula unit. The octahedral or a sites are distributed with body centered cubic symetry in each octant.

B) The distribution of tetra- and dodecahedral sites is Iong the non-diagonal cube face bisectors of each octant. 
c) The c and d sites are distributed alternately along the mutually perpendicular Eace bisectors so as to define a three-fold axis along $\langle 111\rangle$.

ig. 4. A) The entire unit cell can be generated from a single octant by considering the non-diagonal face bisectors to be two-fold axes of rotation.

B) By successively rotating the octant cube by $180^{\circ}$ about these axes the partial unit cell can be generated having $<11>$ axes distributed as shown.

C) Continued rotation of $180^{\circ}$ about the octant face bisectors generates the garnet unit cell which has a single unique three-fold axis along $\langle 111\rangle$.

Fig. 5. A) Aisplacement of $\overrightarrow{\mathrm{R}}_{2}=a_{0} / 2<100>$ results in exchanging retrahedral and dodecahedral sites whereas the densely packed b.c.c. octahedral sublattice is unaltered.

B) A displacement of $\left.\vec{R}_{b}=a_{0} / 2<110\right\rangle$ produces similar results ro $\left.a_{0} / 2<100\right\rangle$. Consequently defects in garnets producing these displacements have electrostatic as well as elastic energies due to the exchange of cation positions. C) Displacements of $\vec{R}_{c}=a_{0} / 4<111>$ leave garnet octahedral sites conserved but alter the distribution of tetrahedral and dodecahedral sites so that formerly unfilled positions becone occupied. This results in quite different elastic and electrostatic energies associated with defects producing 2/4<11> displacements. 
Fig. 6. The increased resolution of dislocations in CGGG using high order bright field and weak-beam dark field imaging techniques is seen in and $C$ respectively as compared with the conventional bright field micrograph of $B$. The diffraction conditions used are inset in each. Improved resolution of KOBF and WBDF images is invaluable in simplifying the interpretation of contrast from defects in CGGG.

Fig. 7. A) A symmetric [001] diffraction pattern from CGGG shows the existence of $\{200\}$ as well as $\{400\}$ cype reflections. $B$ ) and C) Upon tilting the crystal to excite either of the two mutually perpendicular systematic rows of $n\{400\}$ $(n=$ an integer) reflections the $n\{200\}$ reflections disappear. This is consistent with double diffraction phenomena and can be explained in terms of interactions between $\{400\}$ and $\{420\}$ reflections.

Fig. 8. Two closely spaced chemical defects about $0.25 \mu$ in size are seen as dark oval areas against a lighter CGGG matrix in these $B F$ images. In the sequence $A B C D$ the specimen was rilted to make the diffraction vector $\vec{g}$ rotate clockwise. The lobed contrast distorts to "follow" the operating reflection in a maner typical of strain contrast. Qualitative analysis of these strain effects, accounting for the overlap of strain Fields from the two closely spaced defects, shows these are each ellipsoidal in shape. 
Fig. 9. Microsegregate defects in CGG axe conmonly $0.2-0.64$ in sige and consist of a dark halo surrounding a light circular patch. The defect/matrix interface is quite sharp and appears curved in this bright field image.

Fig. 10. Amicrosegregate in a thin area of a CGGG specinen is imaged in bright field. The light central patch is seen to intersect the specimen surfaces leaving the inner annulus $S$, and reaches its widest equatorial dimension at M near the middle of the foil. This is consistent with this feature being spherical in shape. Since the electron beam is transmitted undeviated through the inner annulus $S$, the sphere is believed to be hollow with its spherical morphology maintained by the hydrostatic pressure of oxygen gas. Type 1 dislocations are seen in contact with the interface at the regions designated d.

Fig. 11. The chemical defects both with and without central spheres appear as dark areas against a lighter matrix in both bright field and dark field, as seen in $A$ and B respectively. This indicates that electrons undergo greater absorption in these areas and can be atcributed to variations in composition and occupancy of garnet cation sites.

Fig. 12. Microsegregates with and without central spheres are comonly interspersed in linear arrays along $<100\rangle$ directions as seen in this pair of bright field micrographs from adjacent areas in a [001] oriented specimen. Note the split dislocarion ring contrast of region $S$ in Fig. $12 \mathrm{~A}$ having a line of no 
controst perpendicular to $\vec{g}$. The region $\mathrm{L}$ of $\mathrm{Fig} .12 \mathrm{~B}$ shows strain field overlap among defects not having developed central spheres. The features marked $\mathrm{k}$ are cracks.

Fig. 13. STEM/EDAX spectra showed the segregated regions to be rich in gallium and depleted in germanium relative to the CGGG marrix. This schematically shows that the spectra were obtained by first placing a $200 \mathrm{~A}$ diameter probe in the matrix near a defect and comparing the spectrum generated with another obtained by placing the probe on the segregated region.

Fig. 14. Actual spectra obtained from matrix and defect are seen in $A$ and $B$ respectively. The $K_{\alpha}$ peaks are marked and show that only elements intrinsic to $\mathrm{Ca}_{3} \mathrm{Ga}_{2} \mathrm{Ge}_{3} \mathrm{O}_{12}$ were found, except for $\mathrm{Fe}$ and $\mathrm{Cu}$ which arise from the ixon pole pieces of the microscope and copper grid upon which the specimen was mounted. Oxygen was not detectable using this technique.

Fig. 15. Type 2 dislocation lines lying in the matrix of a $\langle 120\rangle$ oriented specimen are seen in region of the bright field images of $A$ and $B$. The dislocations are out of contrast in $C$. These dislocations are mixed in nature having Burgers vectors parallel to $|113|$ and line direction of $|210|$.

Fig. 16. A microsegregate with a sphere in a [001] oriented CGGG specimen is imaged in three different difracting conditions. In A, two mutully perpendicular Type 3 dislocation loops are seen to surround the segregate while in each of $B$ and C the crystal was tilted to make each of the loops go out 
of contrast in turn. Trace and $\vec{g} \cdot \vec{b}$ analyses show these loops to be of edge type having Burgers vectors along the <100\$ directions nomal to the $\{100\}$ planes on which the loops lie. Notice that the loops extend beyond the segregated region and into the natrig naterial indicating that climb has occurred. The feature $\mathrm{K}$ is a crack.

Fig. 17. In this WBDF micrograph an isolated segregate in a $<120>$ oriented specimen has been imaged and the spherical void and dislocation loops on $\{100\}$ planes are visible. Note the two dislocation loop images in the region marked d. It is suspected that this is not a double image of a single loop because of the weak beam conditions used to form the image. Instead this is evidence of either two concentric and coplanax perfect dislocation loops or a single dislocation loop which has dissociated into partials lying on a $\{100\}$ plane.

Fig. 18. A mechanism proposed by Matchews for growth of single (or multiple) large loops around inclusions in gadolinium gallium garnet reguires a small prismatic loop outside the inclusion to grow by climb according to the sequence seen in $A, B$, and $C$.

Fig. 19. Matthews mechanisn for formation of pairs of loops around chemical inhomogeneities calls for a small prismatic loop "a" to be nucleated. It grows by absorbing point defects until the configuration $b$ is reached. Continued climb permits the crescent shaped loop to encircle the inclusion until 
intersecting sections annihilate upon contact on the other side of the inclusion, leaving two concentric loops $C_{1}$ and $\mathrm{C}_{2}$ having the same Burgers vectors.

Fig. 20. Smal1 loop-like precipitates $0.1 \mu$ or less in size are imaged in high order bright field and weak beam dark field in A and 8 respectively. Evidence of coalescence of several. closely spaced precipitates can be seen in region $\mathrm{C}$. The pocked appearance of the matrix shows black/white contrast dependent on $\vec{g}$ and the mode of imaging (i.e. BF or DF), indicative of point defect clusters.

Fig. 21. Wide images due to surface strain relaxation from small disc-shaped precipitates located near the specinen surface can be seen in this BF image. Further support that the pocked background is due to point defect clusters can be gained by observing their contrast reversals across extinction contours.

Fig. 22. The small disc-like precipitates in a [001] oriented specimen are seen Eace-on as well as edge-on in this BF image. Trace' analysis reveals the precipitates to lie on $\{001\}$ planes. The size and contrast (i.e. line of no contrast LC perpendicular to $\overrightarrow{\mathrm{g}}$ ) indicate these to be coherent precipicates with resulting lattice displacenents normal to their plane.

Fig. 23. STEM/EDAX spectra failed to differentiate between the composition of the matrix material near a small disc-shaped precipitate (A) and the precipitate itself (B). However, no traces 
of elements extrinsic to $\mathrm{Ca}_{3} \mathrm{Ga}_{2} \mathrm{Ge}_{3} \mathrm{O}_{12}$ (except the usual Fe and $\mathrm{Cu}$ ) were found, indicating these defects to be intrinsic to the CGGG system.

Fig. 24. Small loop-like precipitates $0.1 \mu$ or less in diameter are seen in the vicinity of the larger $(0.5 \mu)$ microsegregates, some of which show evidence of the light spheres displaced From their centers. Note the denuded region on either side of the larger microsegregates, suggesting the small disclike precipitates to be a preliminary stage in the formation of the larger chemical defects. The perspective in the BF image is along [001]. 
A

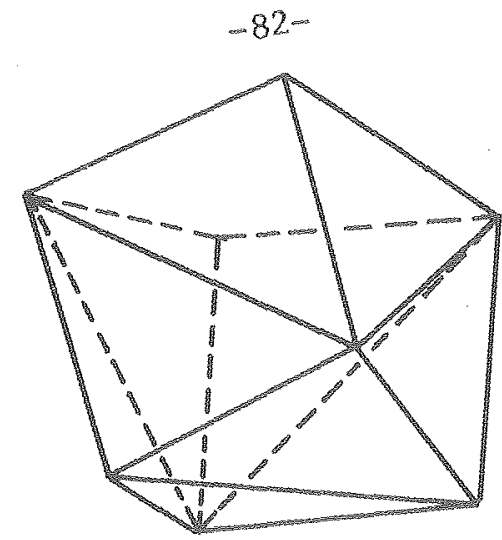

8

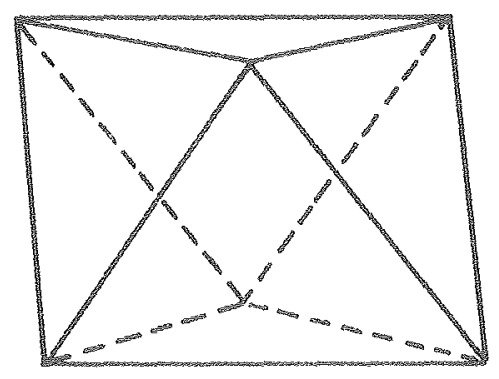

C

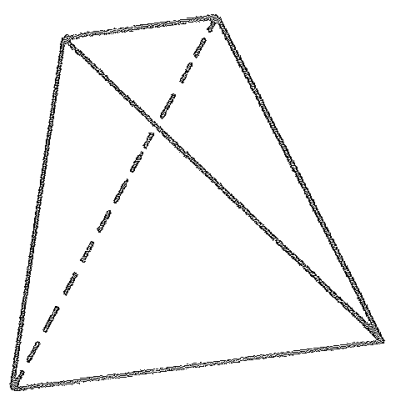

XBL. $788^{-5603}$

Fig. 1 

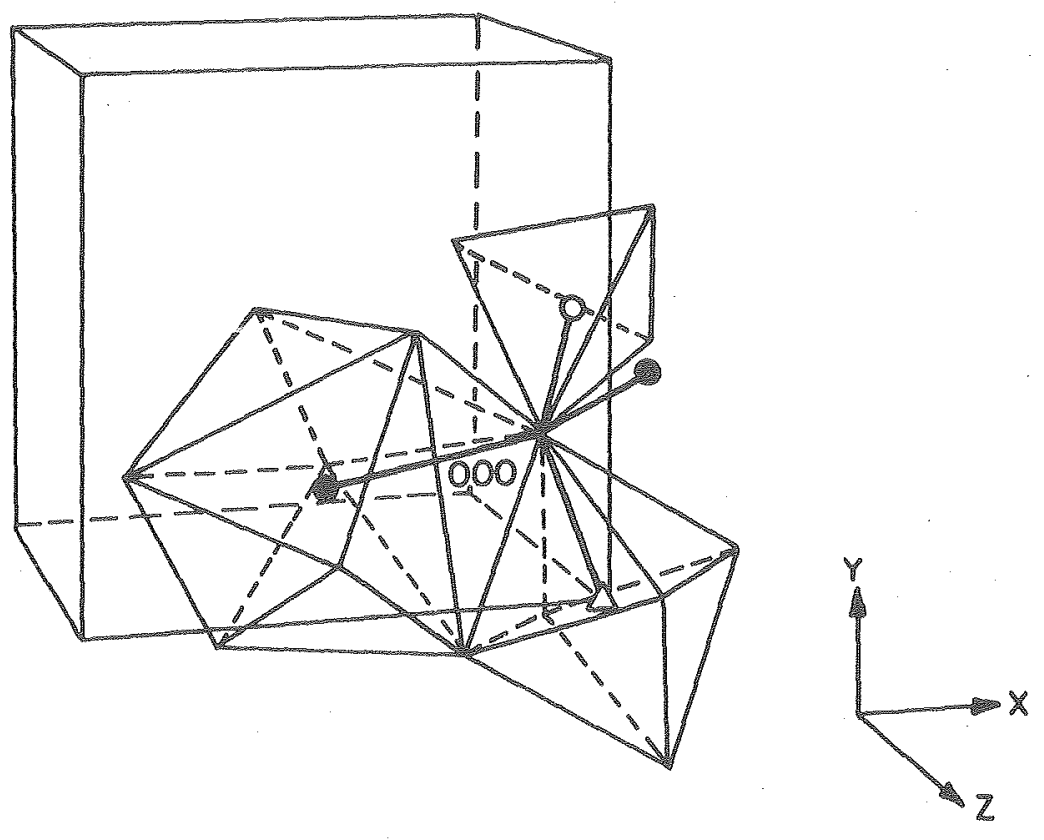
$\Delta G^{3+}(0)$ of $\left(00 \frac{1}{2}\right)$

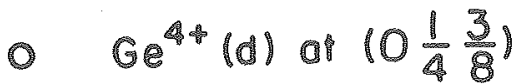
- $\mathrm{Co}^{2}(\mathrm{c})$ of $\left(-\frac{1}{4} \frac{1}{8} \frac{1}{2}\right)$ and as $\left(0 \frac{1}{4} \frac{5}{8}\right)$

Fig。 2 
A

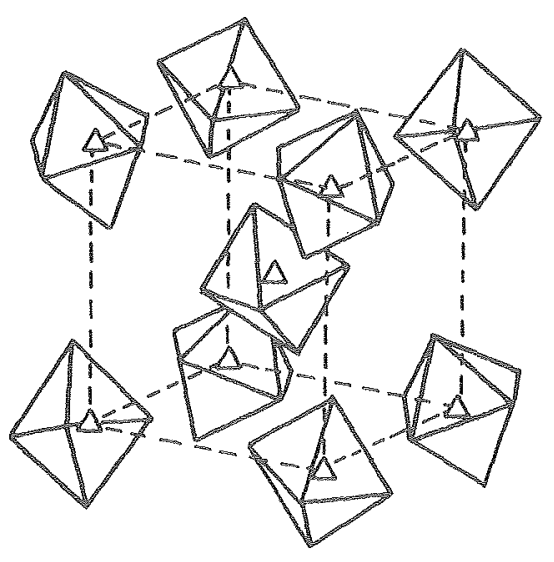

B

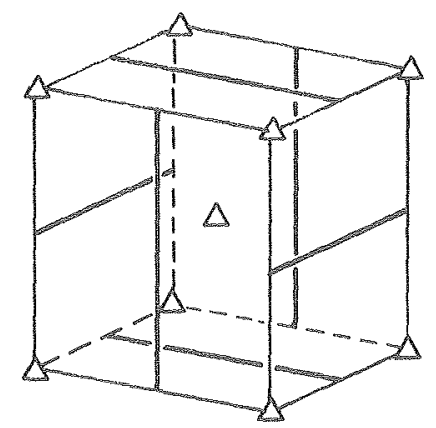

C

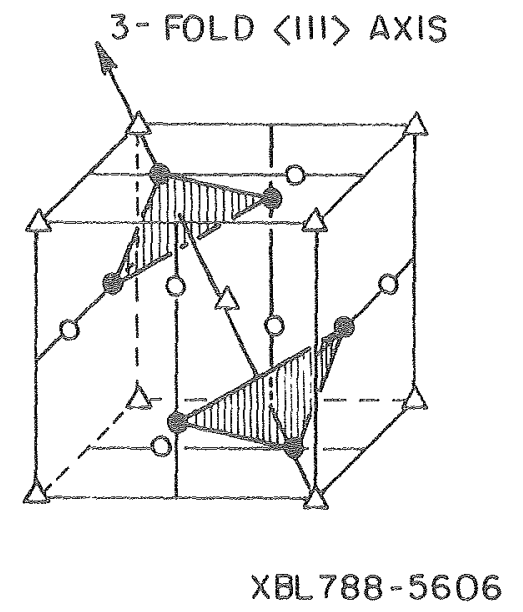

FIg. 3 
$A$

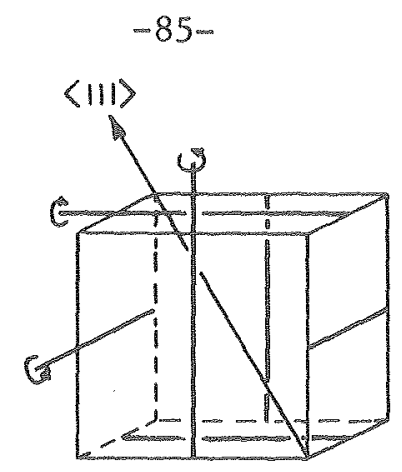

B
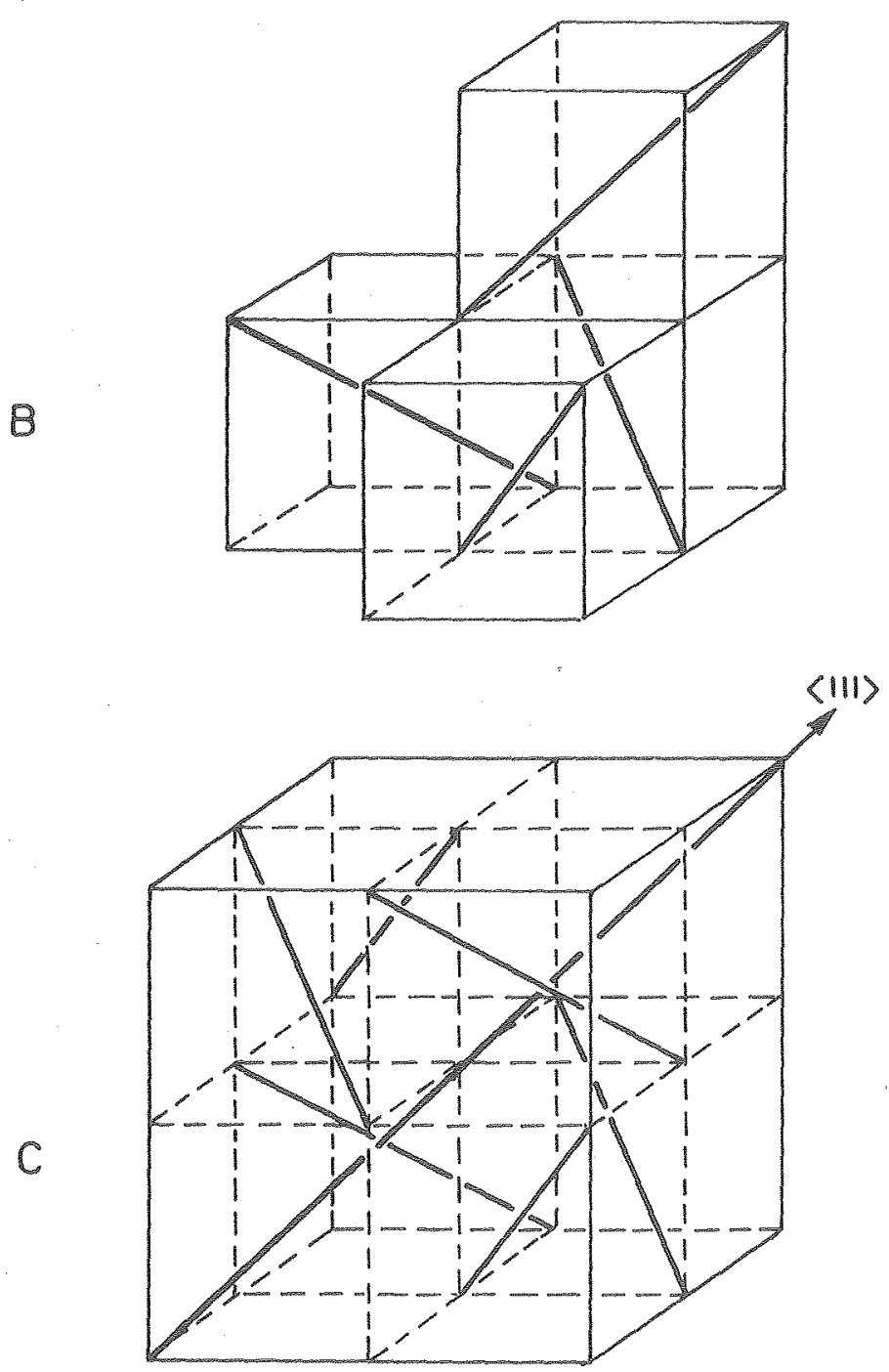

XBL788-5605

Fig. 4 

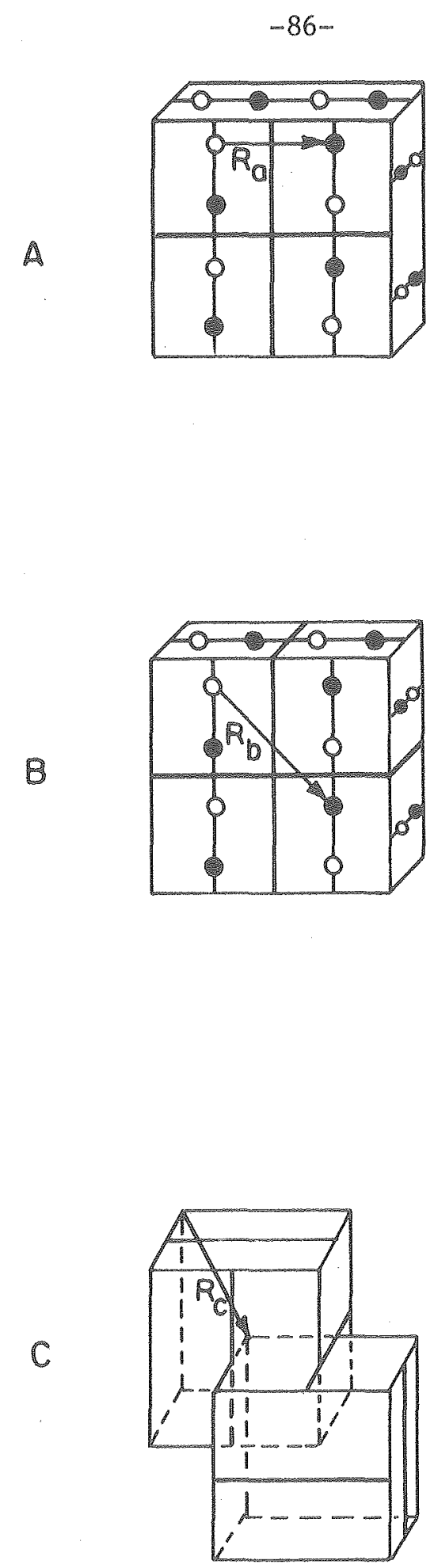

XBL $788-5607$

Fig. 5 

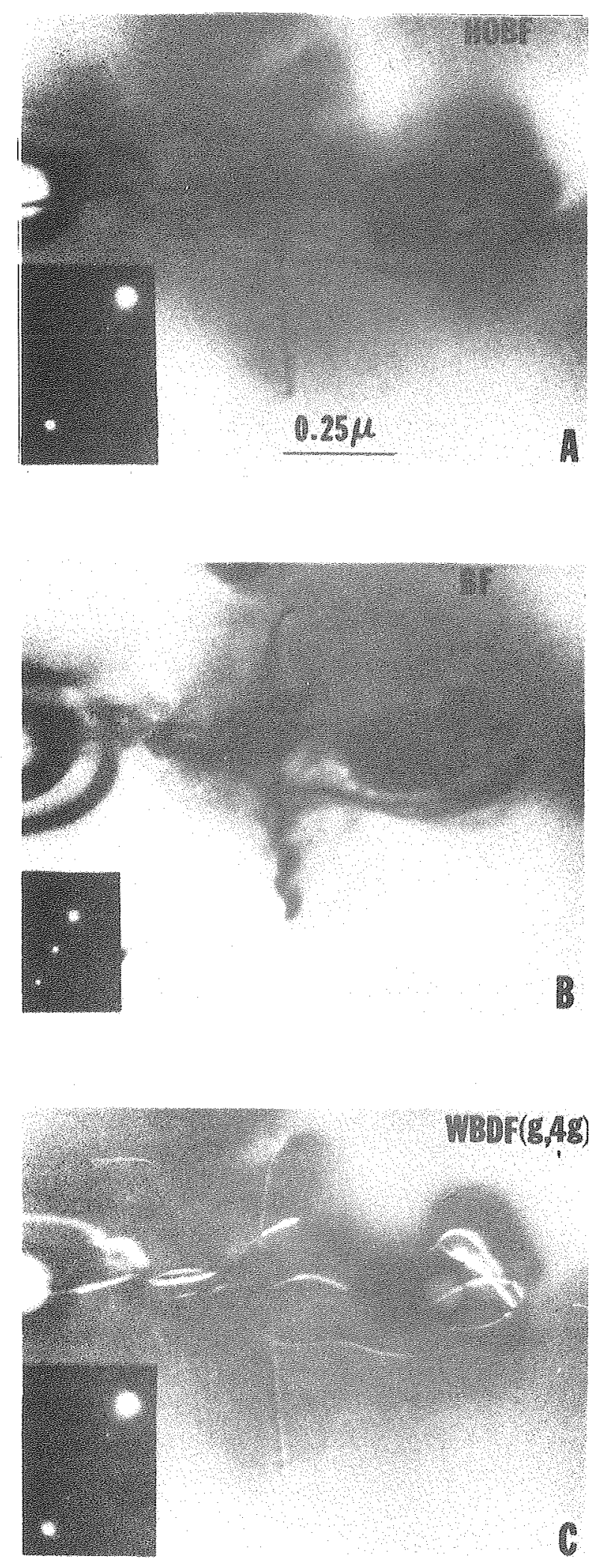

XBB $788-9497$

Fig。 6 

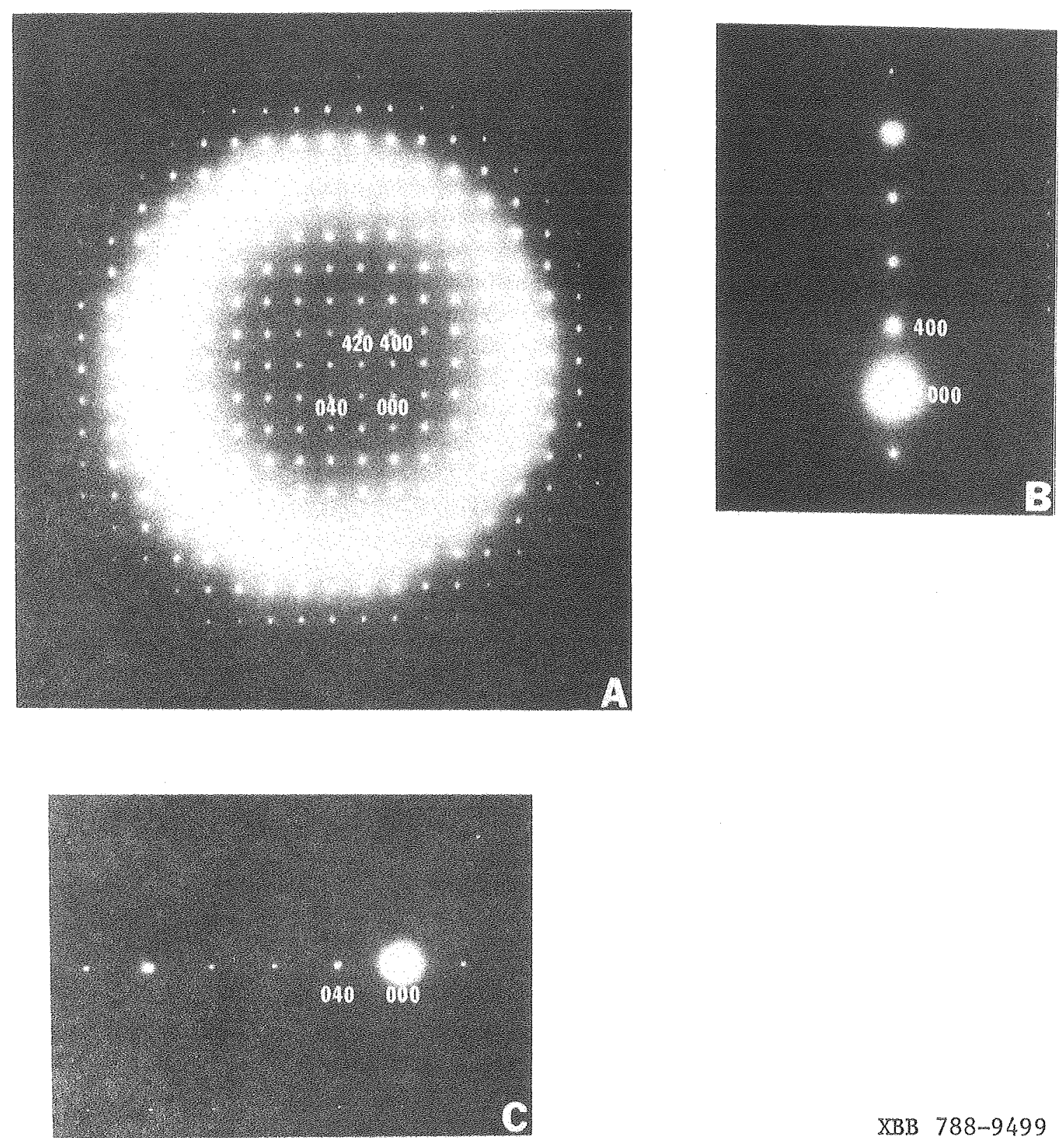

XBB 788-9499

Fig. 7 

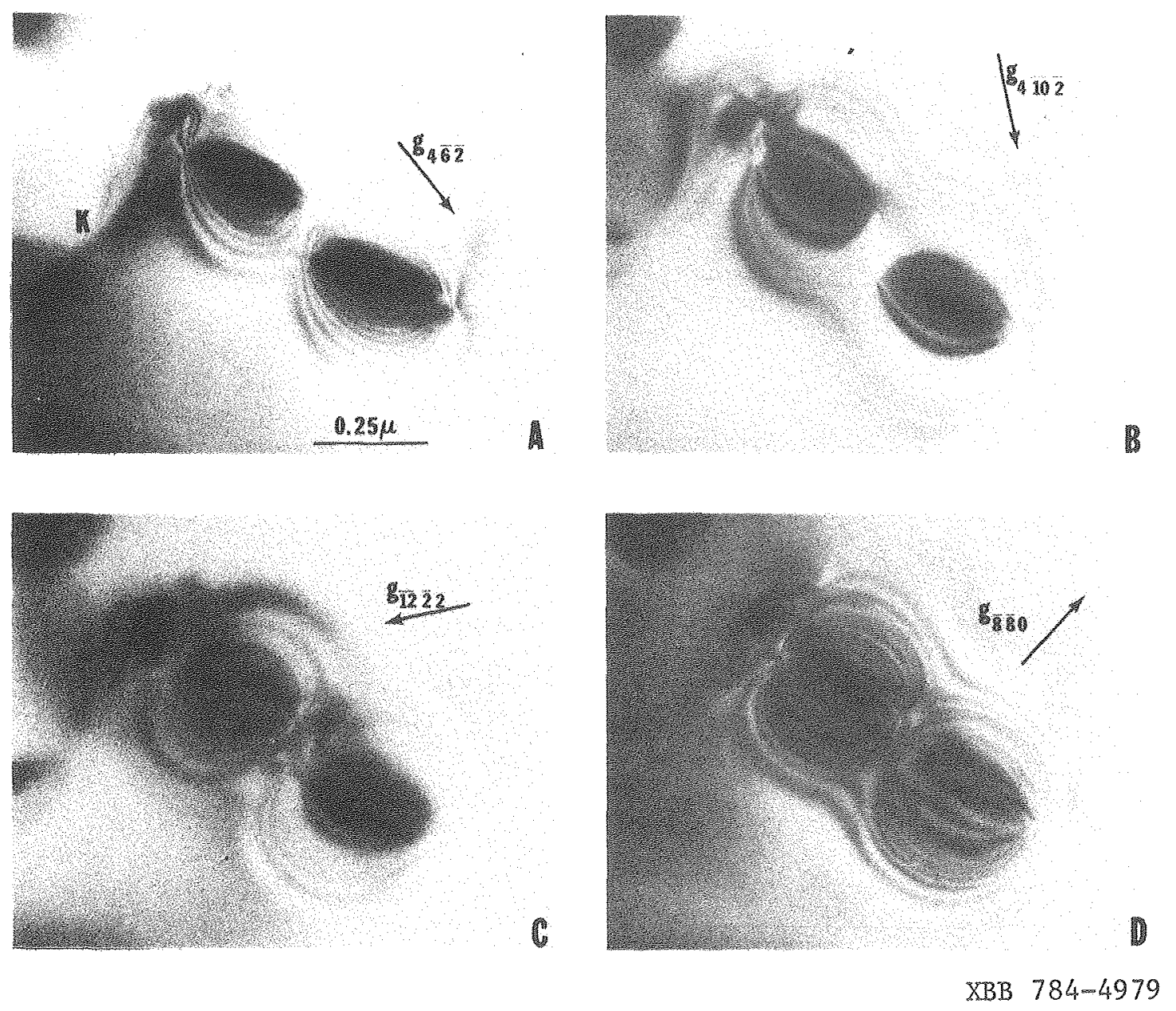

Fig. 8 


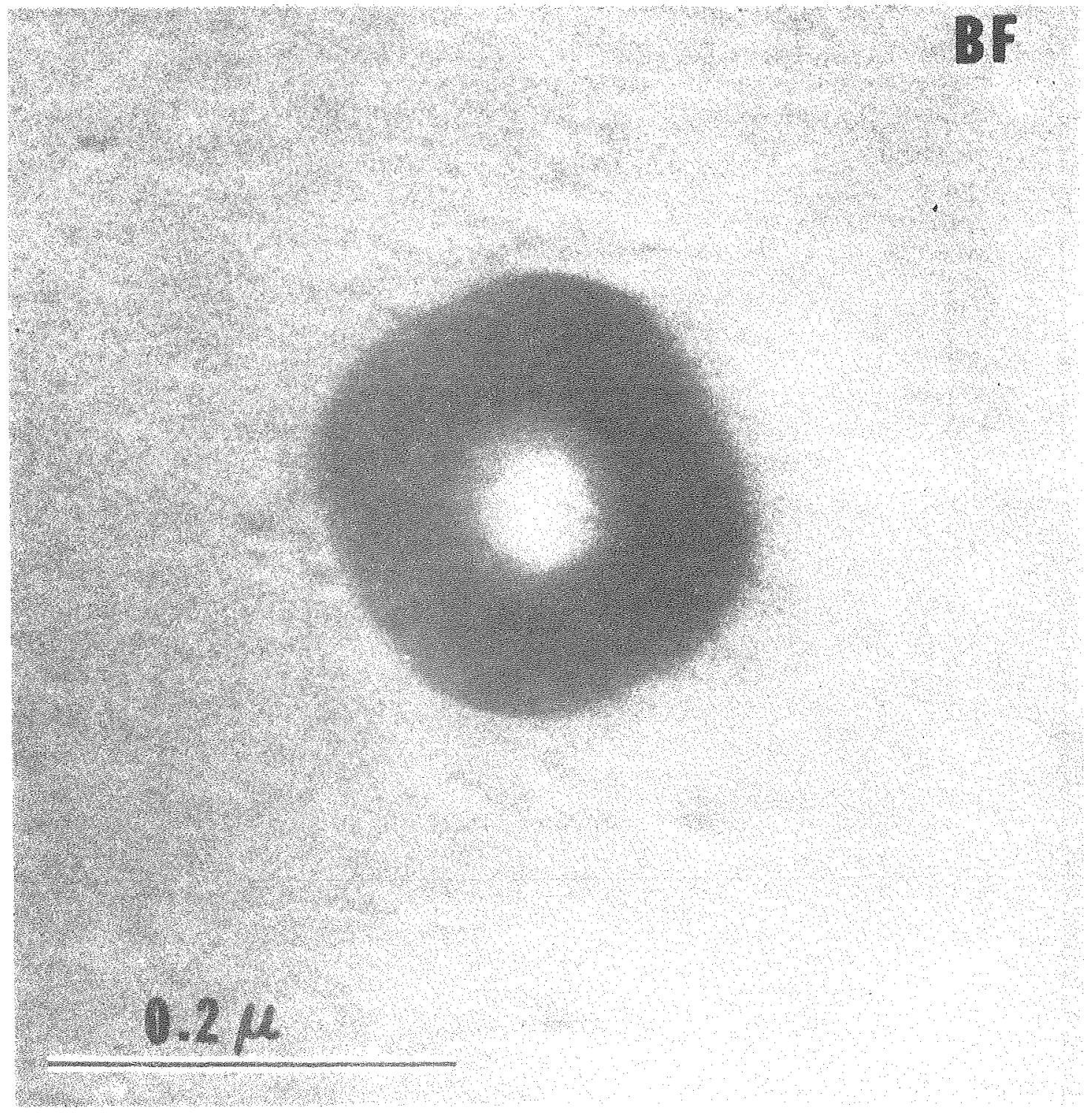

XBB 788-9506

Fig. 9 


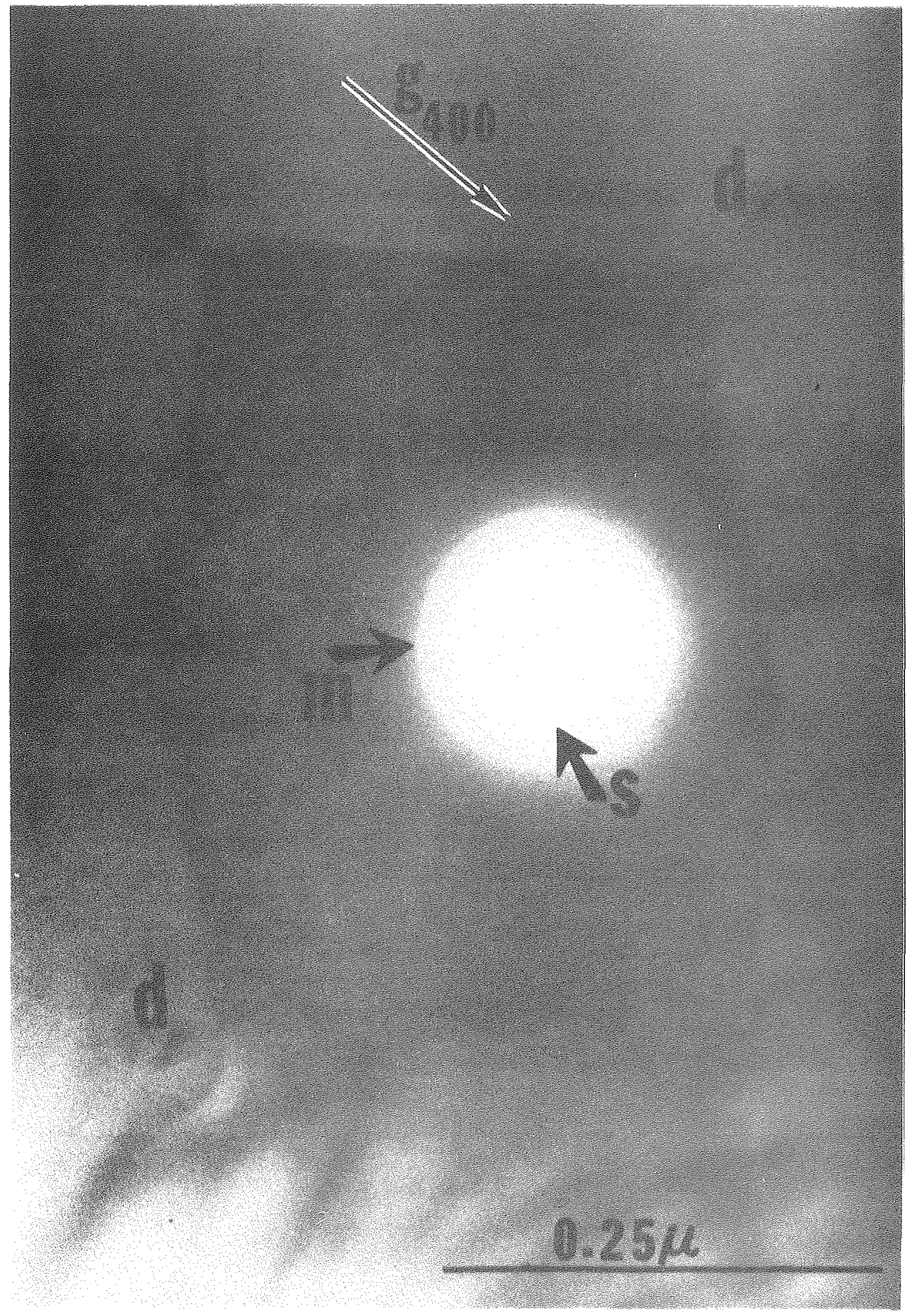

XBB 788-9505

Fig, 10 

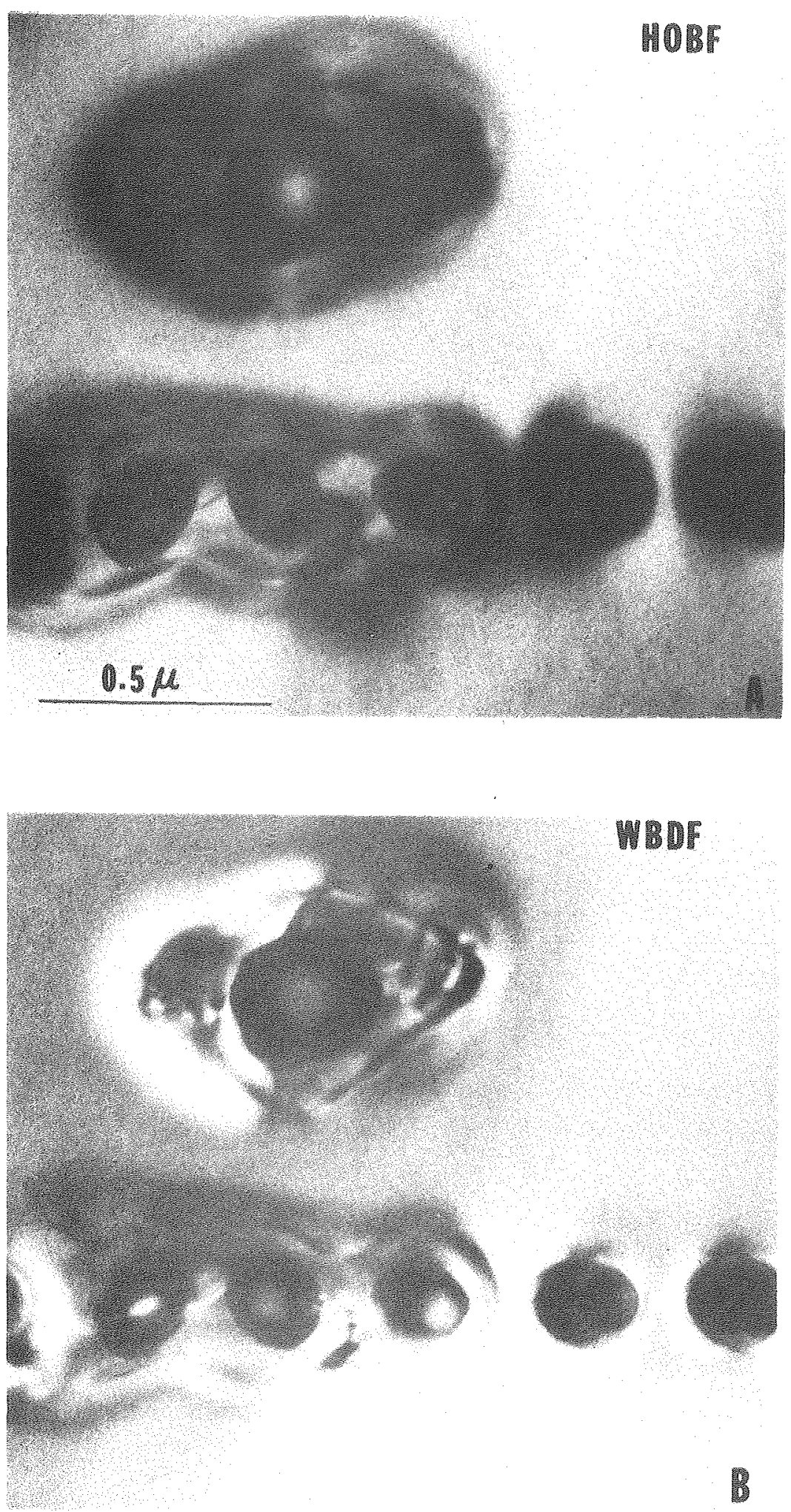

XBB $\quad 788-9500$

F1g. 11 

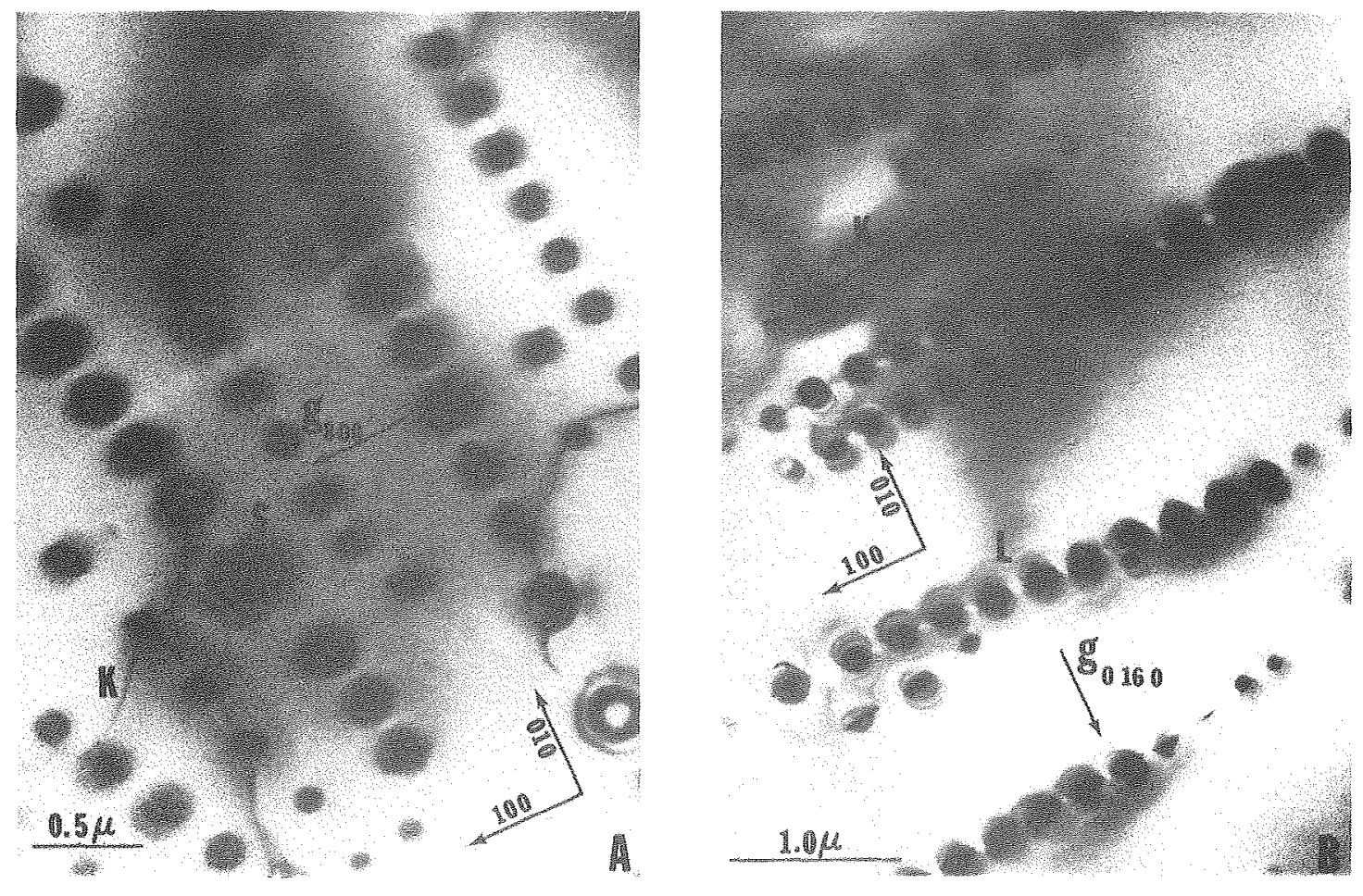

XBB $\quad 788-9496$

Fig. 12 


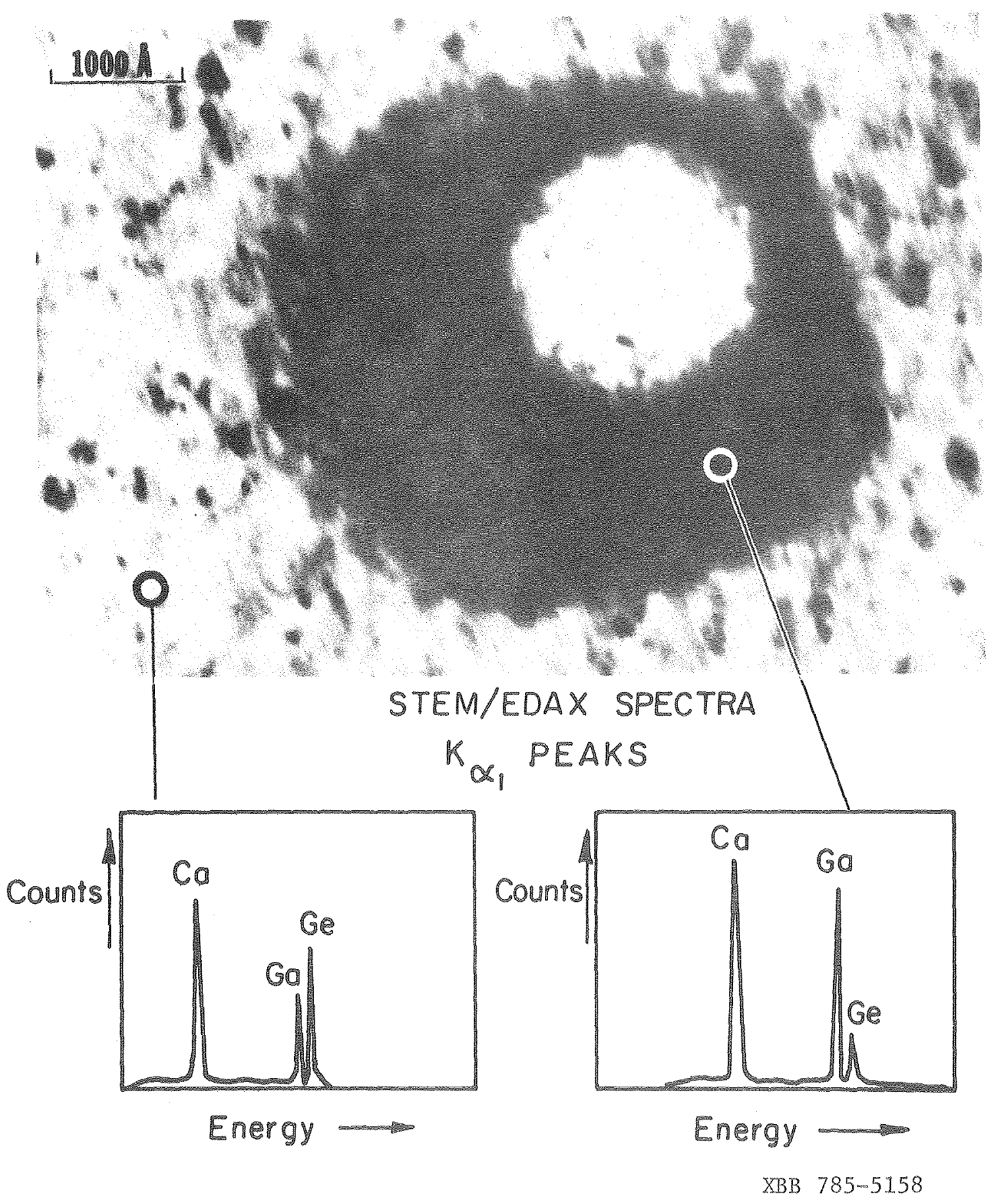

Fig。 13 

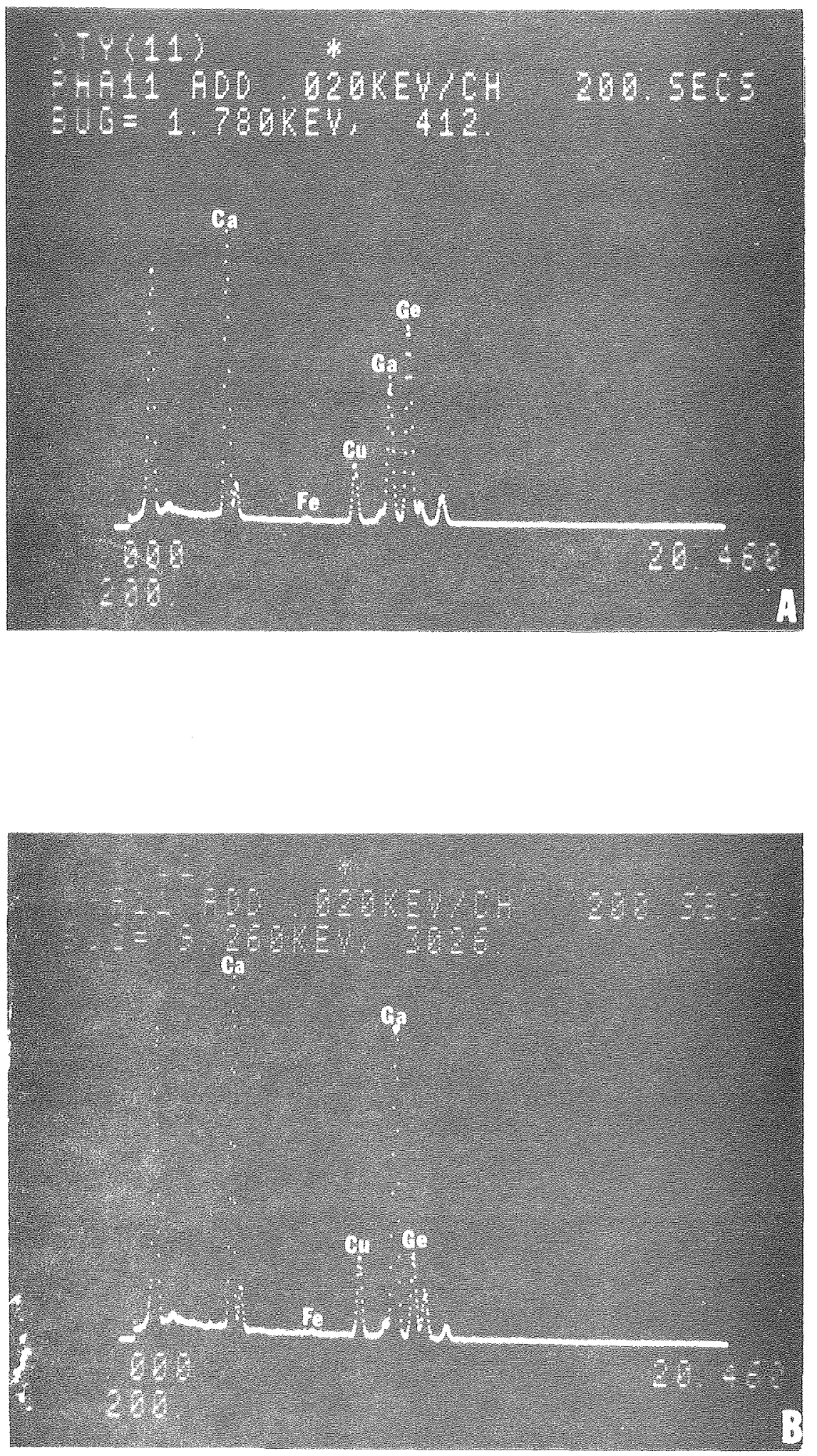

CBB 788-9503

Fig. 14 

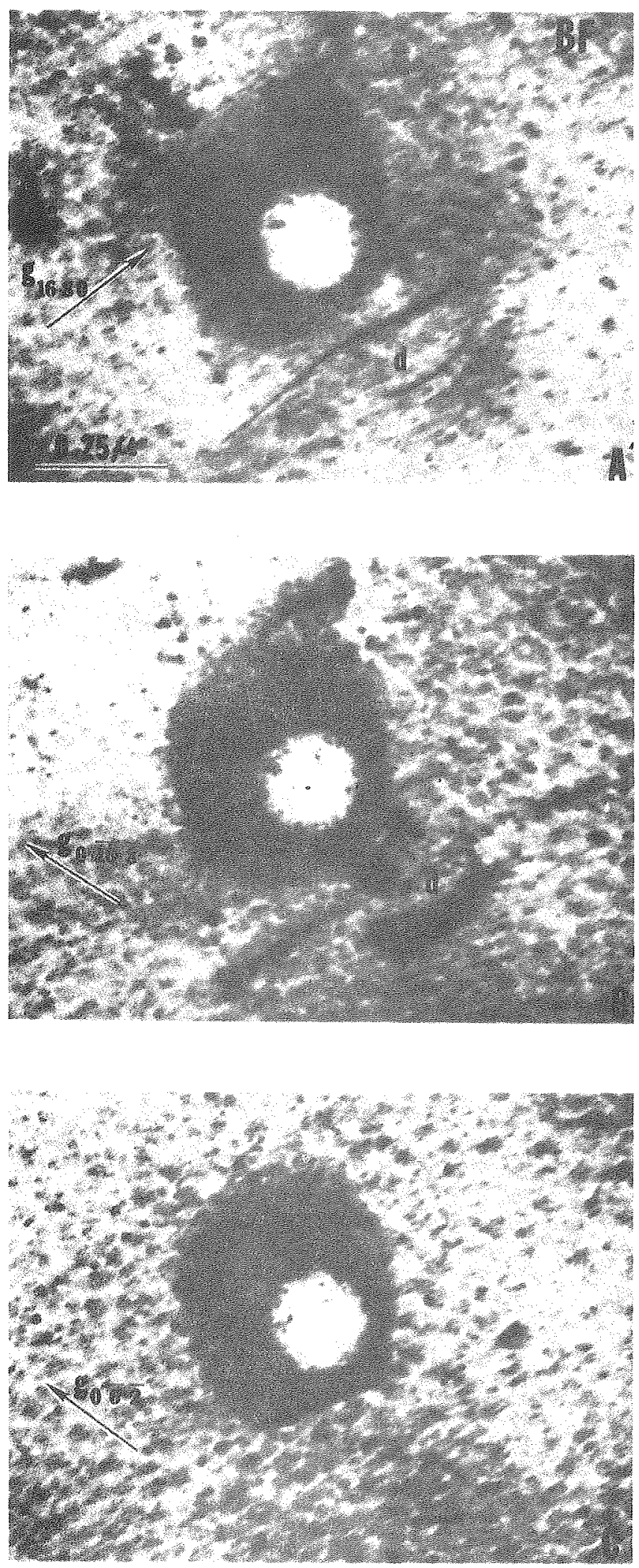

XBB 788-9495

Fig。 15 

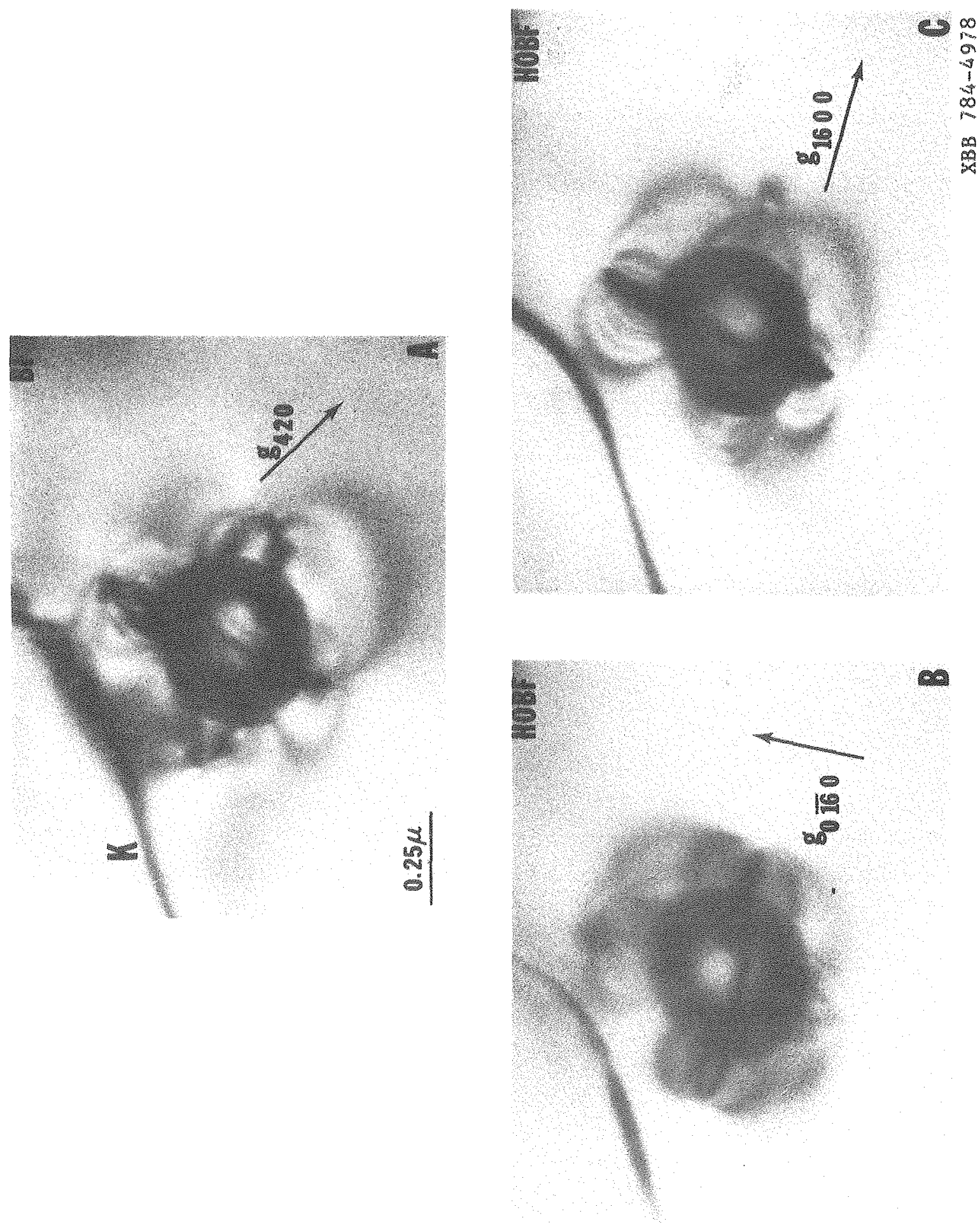

Fig. 16 
$-98-$

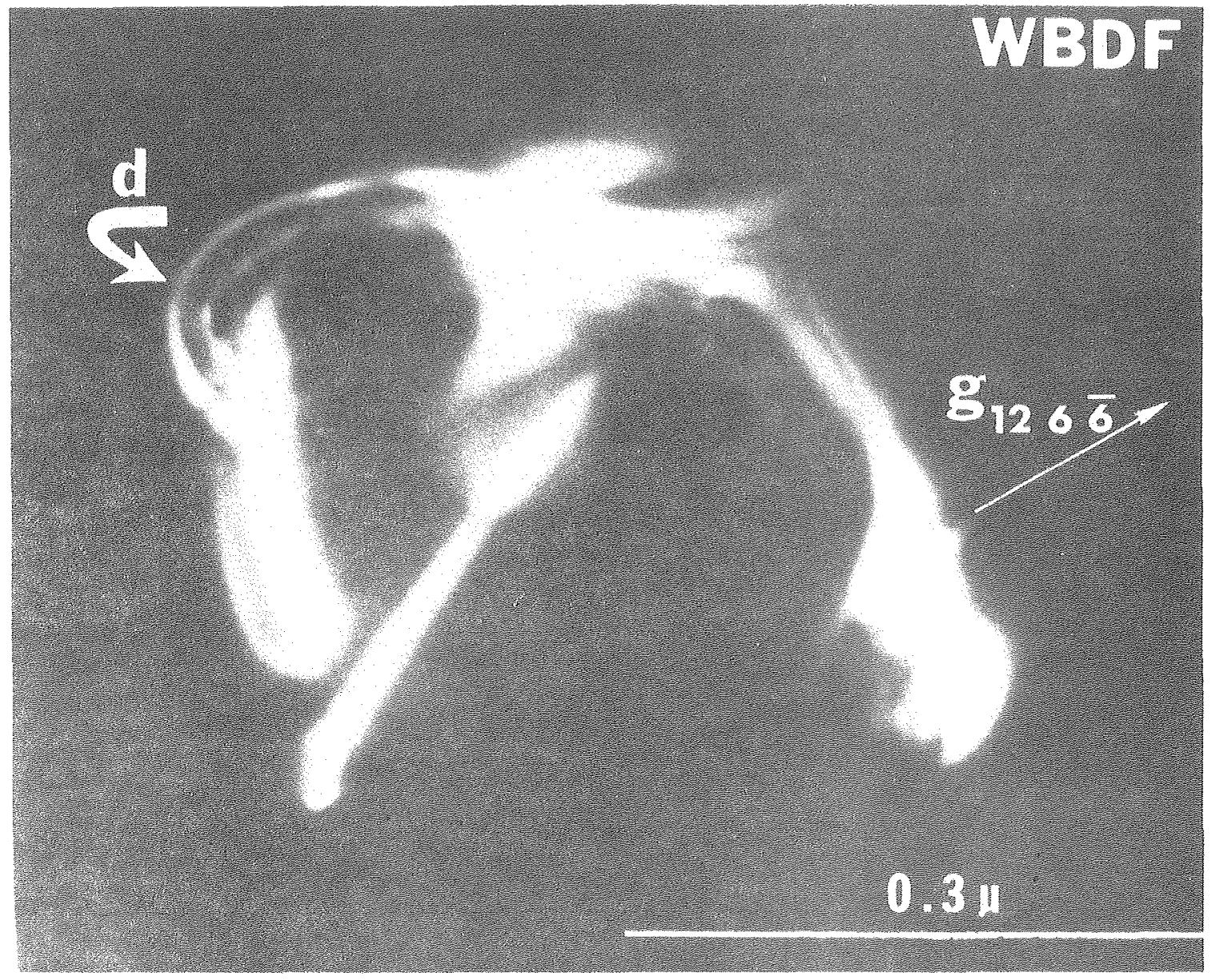

$X B B \quad 784-4976$

Fig. 17 

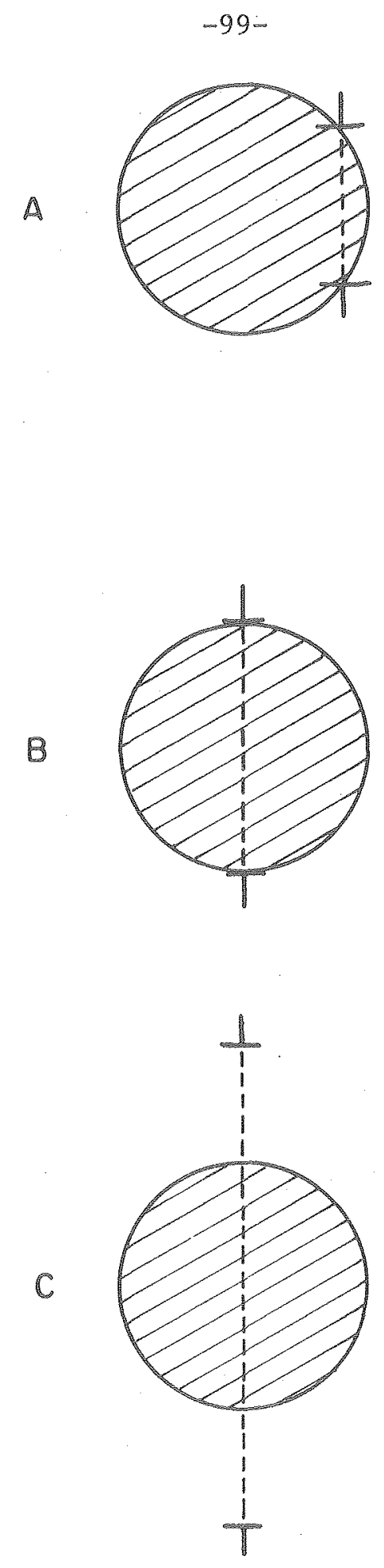

XBL $788-560$

Fig. 18 
$-100$

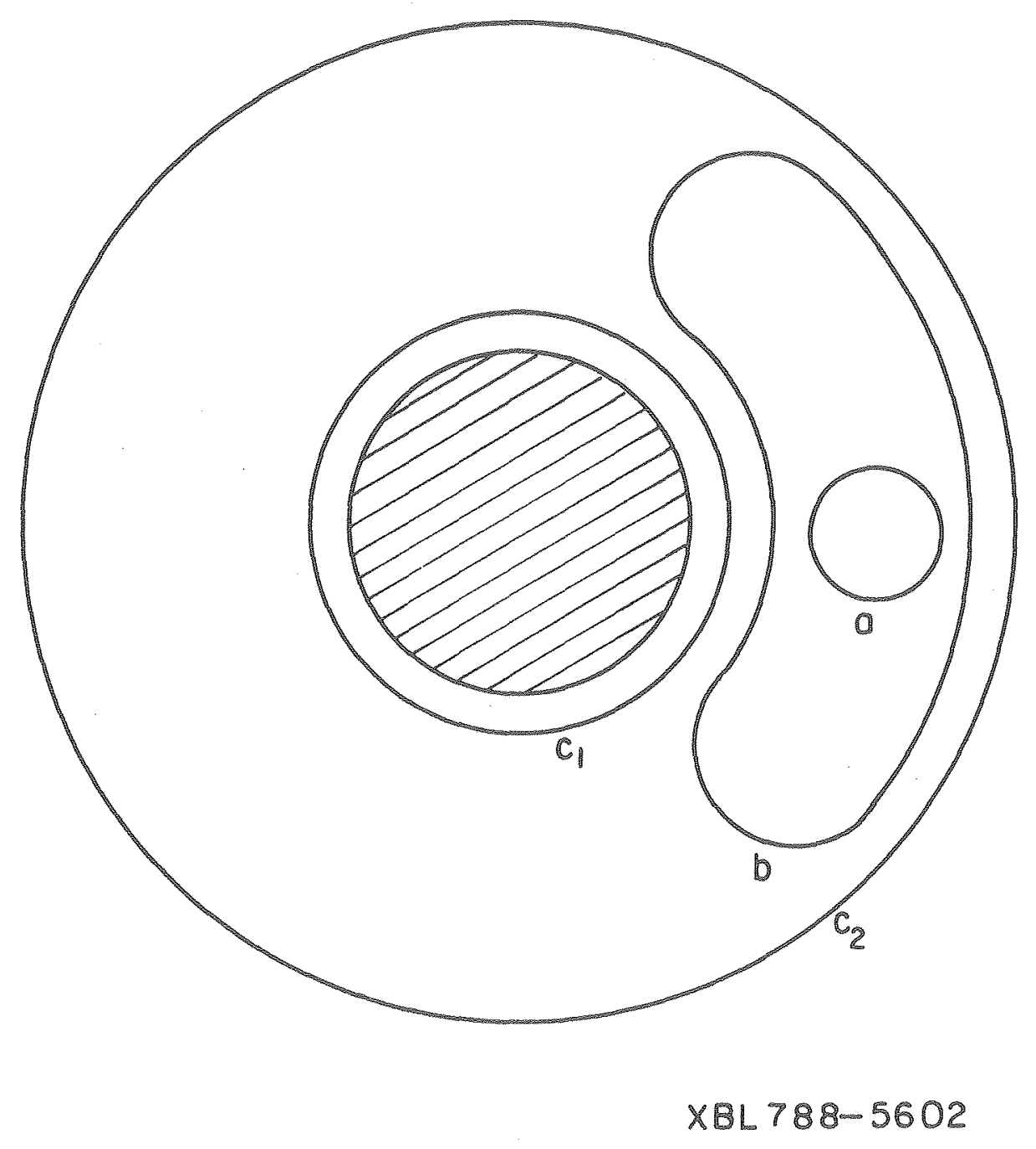

Fig. 19 

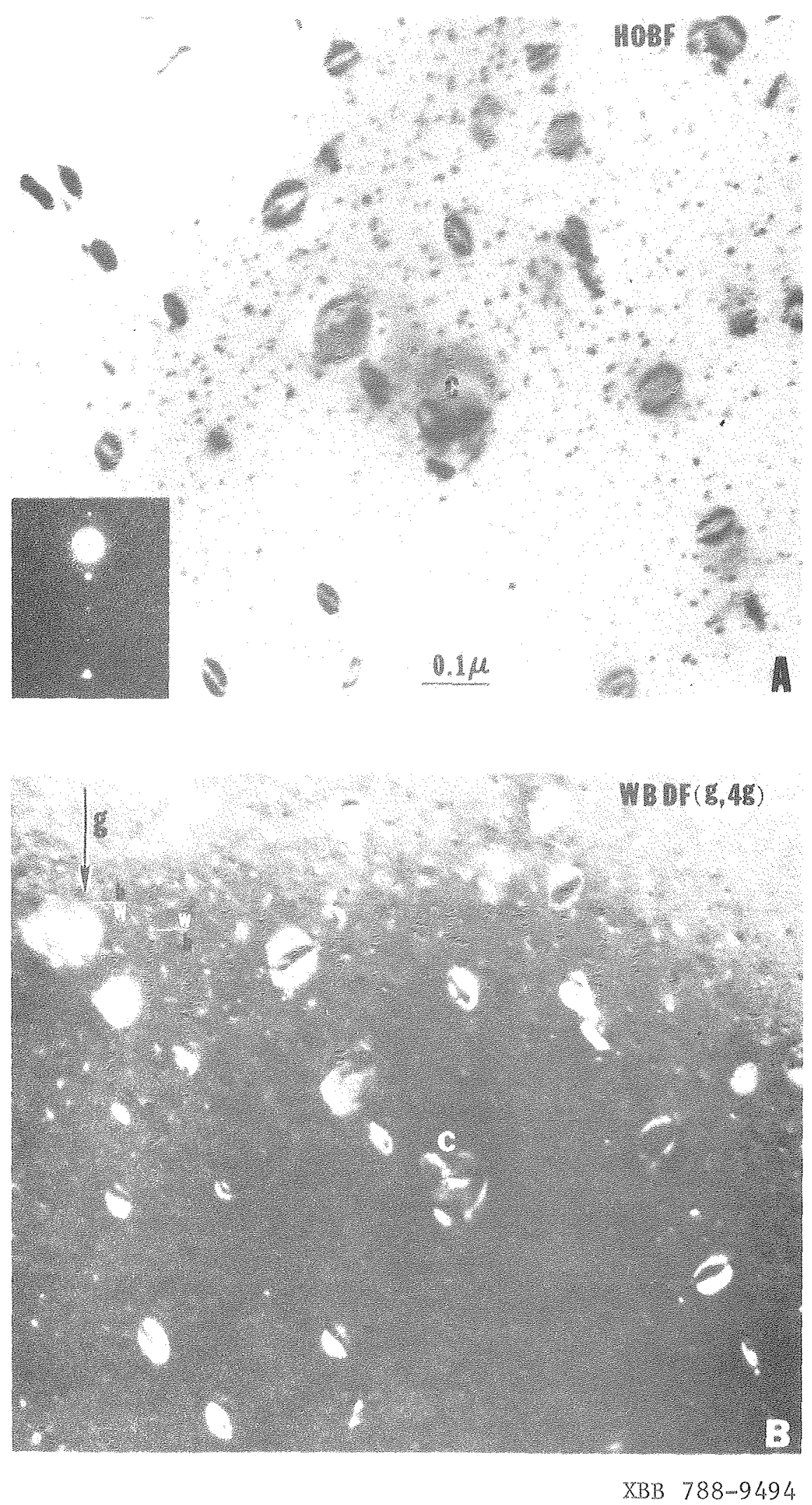

Fig. 20 


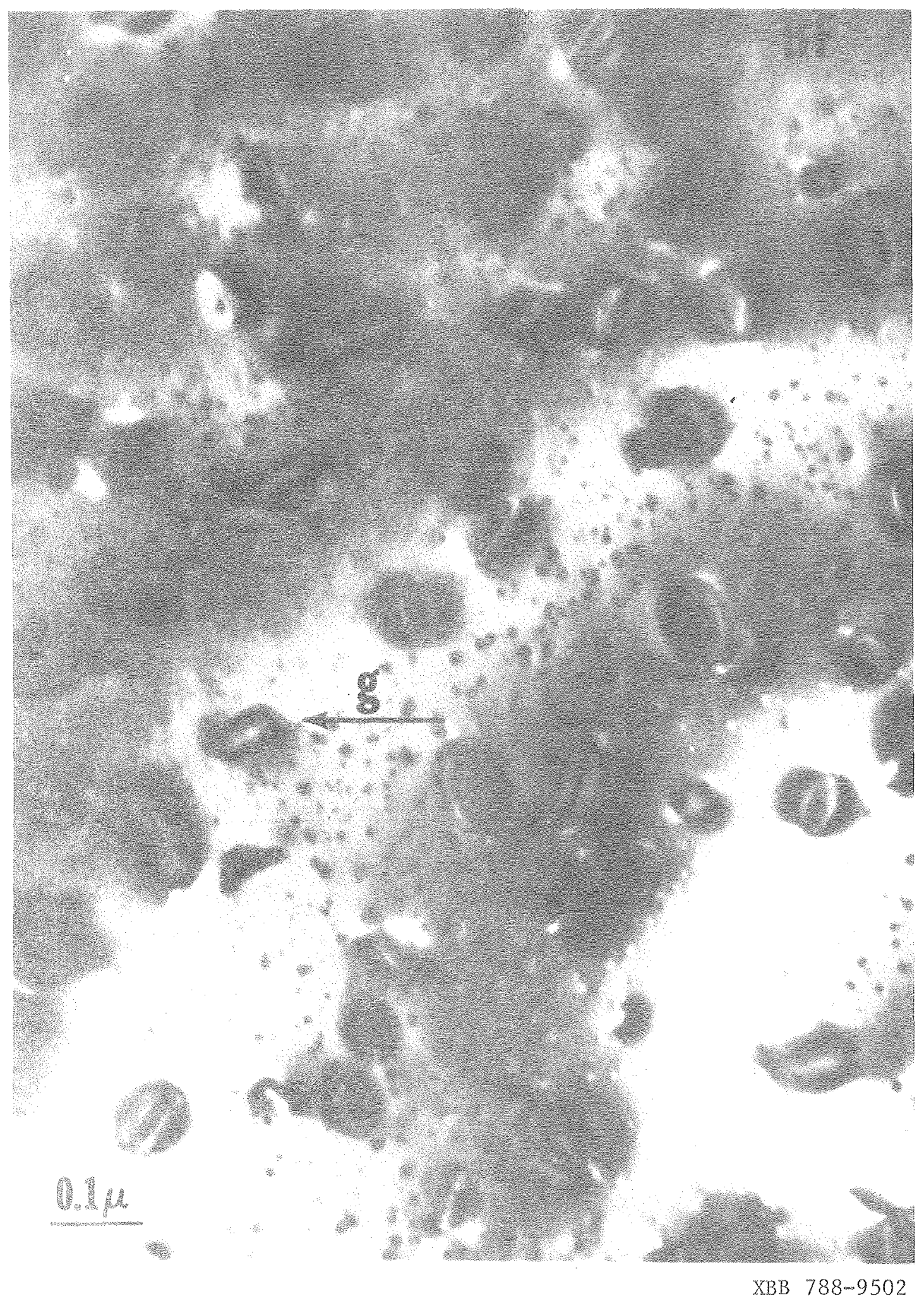

Fig. 21 


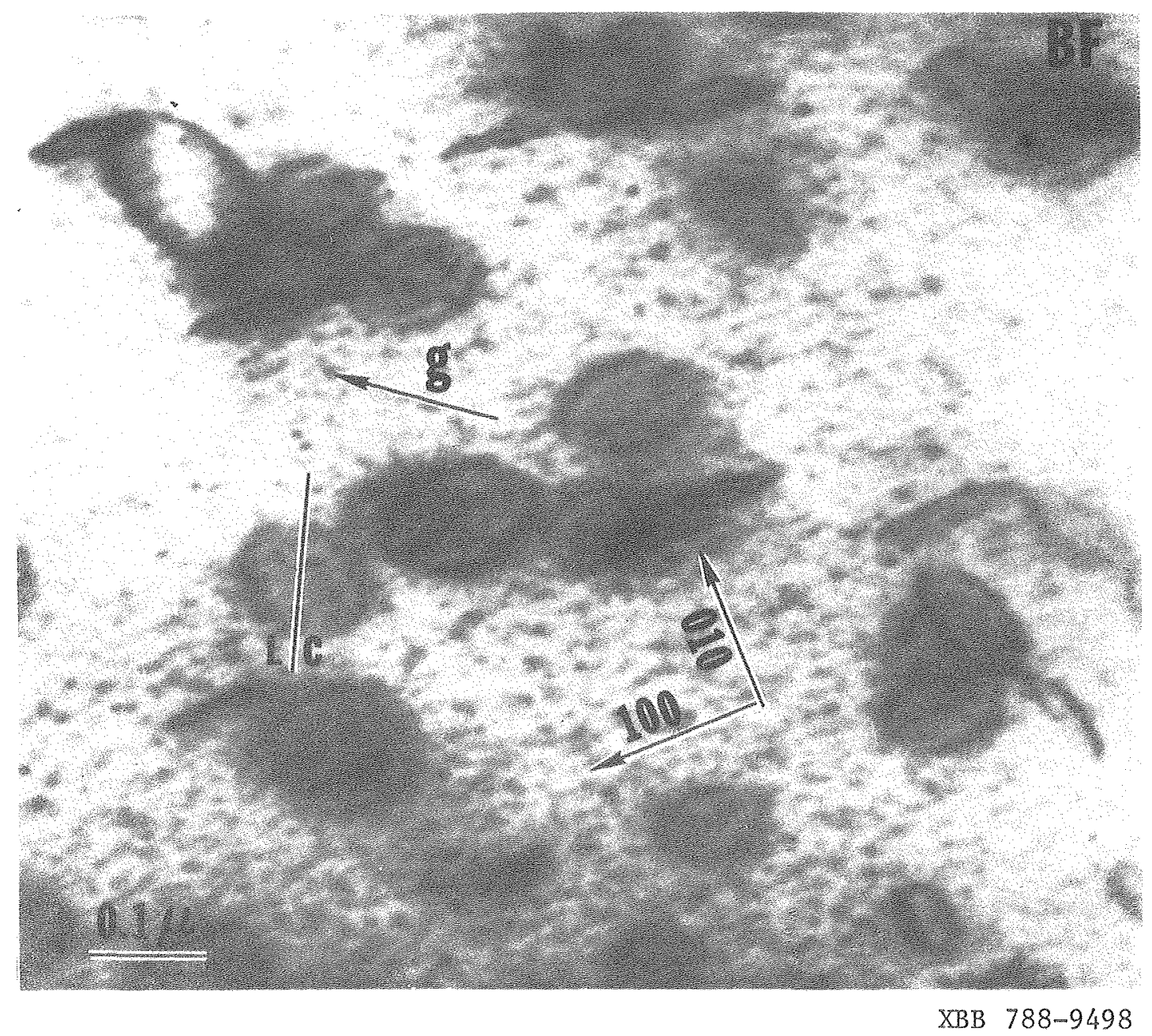

Fig。 22 

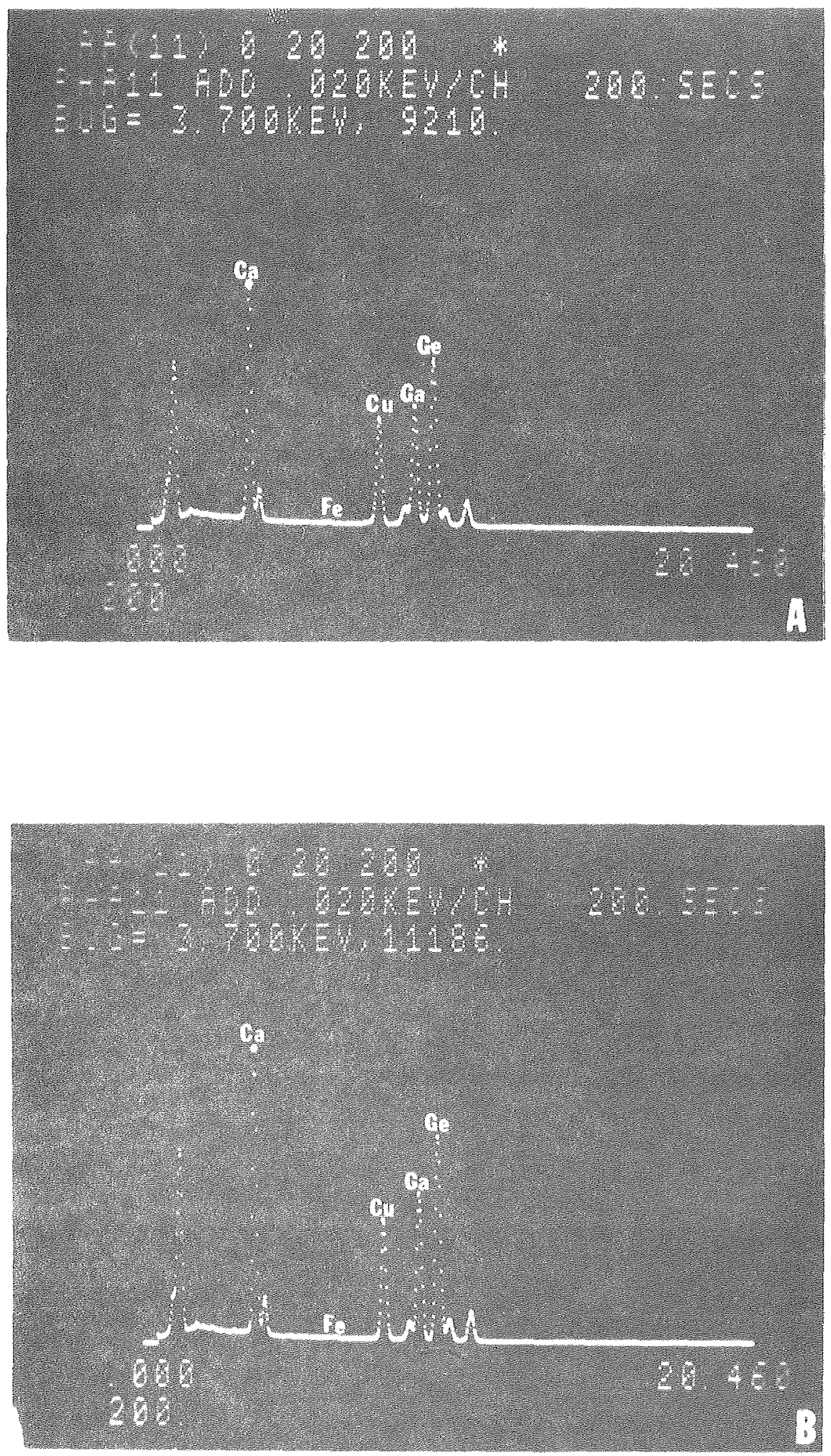

CBB $788-9504$

Figg. 23 
$-105-$

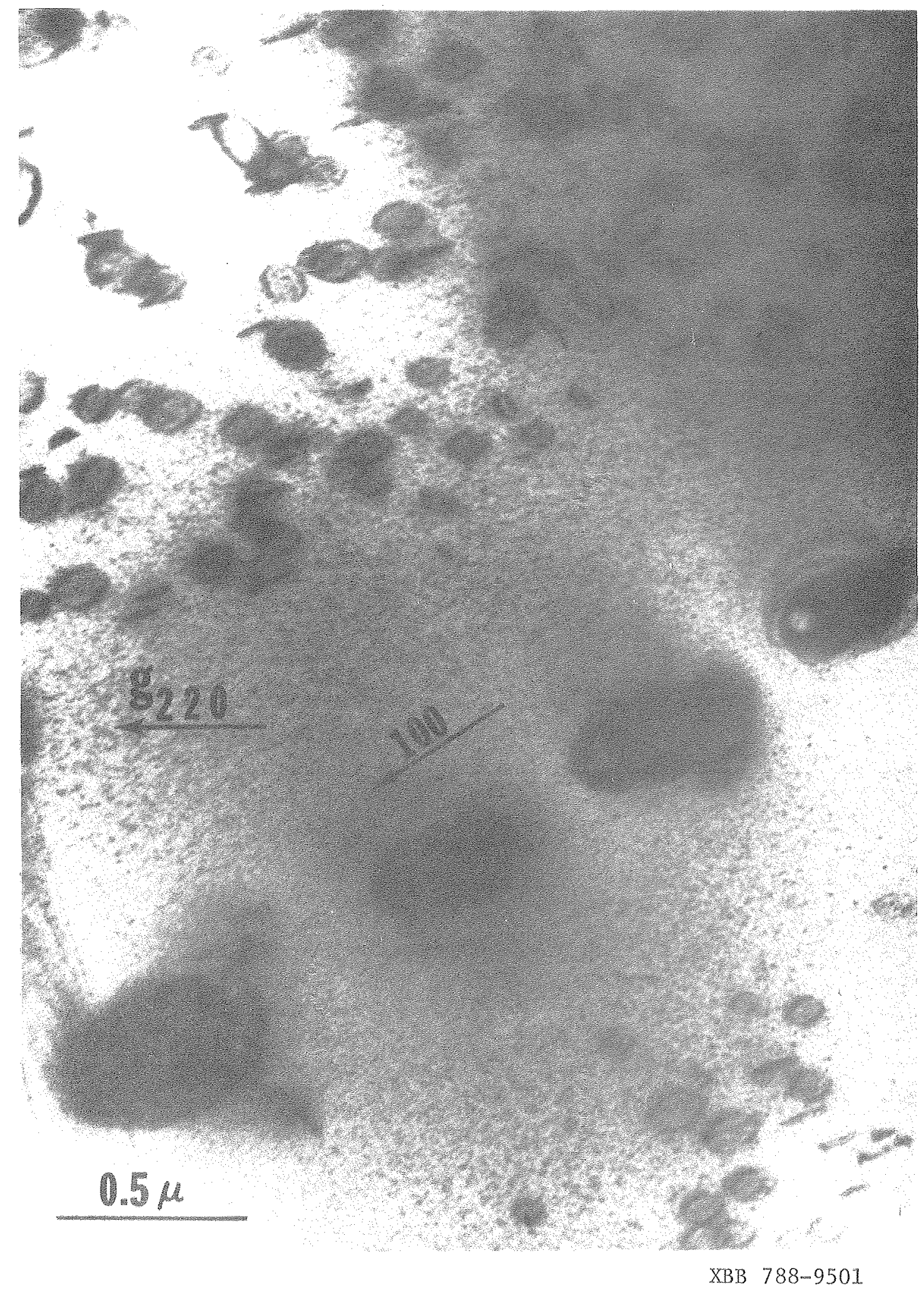

Fig. 24 

This report was done with support from the Department of Energy Any conclusions or opinions expressed in this report represent solety those of the author(s) and not necessarily those of The Regents of the University of California, the Lawrence Berkeley Laboratory or the Department of Energy. 


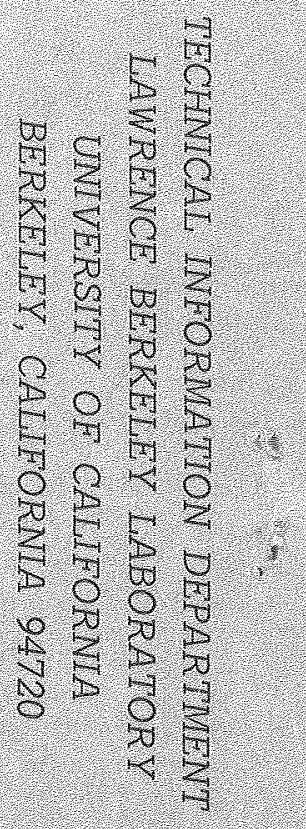

त) 